\title{
A Panoramic Sketch about the Robust Stability of Time-Delay Systems and Its Applications
}

\author{
Baltazar Aguirre-Hernández, ${ }^{1}$ Raúl Villafuerte-Segura $\mathbb{D}^{\mathbb{C}}{ }^{2}$ Alberto Luviano-Juárez $\mathbb{D}^{3}{ }^{3}$ \\ Carlos Arturo Loredo-Villalobos, ${ }^{1}$ and Edgar Cristian Díaz-González ${ }^{1}$ \\ ${ }^{1}$ Departamento de Matemáticas, Universidad Autónoma Metropolitana Iztapalapa, 09340 Ciudad de Mexico, CDMX, Mexico \\ ${ }^{2}$ Centro de Investigación en Tecnologías de Información y Sistemas, Universidad Autónoma del Estado de Hidalgo, Pachuca, \\ HGO, Mexico \\ ${ }^{3}$ Instituto Politécnico Nacional UPIITA, Ciudad de Mexico, CDMX, Mexico
}

Correspondence should be addressed to Raúl Villafuerte-Segura; villafuerte@uaeh.edu.mx

Received 30 May 2020; Revised 20 August 2020; Accepted 5 September 2020; Published 27 September 2020

Academic Editor: Tila Bueno

Copyright (c) 2020 Baltazar Aguirre-Hernández et al. This is an open access article distributed under the Creative Commons Attribution License, which permits unrestricted use, distribution, and reproduction in any medium, provided the original work is properly cited.

\begin{abstract}
This paper presents a brief review on the current applications and perspectives on the stability of complex dynamical systems, with an emphasis on three main classes of systems such as delay-free systems, time-delay systems, and systems with uncertainties in its parameters, which lead to some criteria with necessary and/or sufficient conditions to determine stability and/or stabilization in the domains of frequency and time. Besides, criteria on robust stability and stability of nonlinear time-delay systems are presented, including some numerical approaches.
\end{abstract}

\section{Introduction}

The importance of complex dynamical systems has been increasing dramatically since many real world applications have adopted this behavior. Some of them have become special areas of study such as smart grids [1-5], autonomous vehicles [6-10], biological systems [11-17], distribution networks [18-23], social interaction [24-27], communication systems [28-30], and animal monitoring [31-34], among others.

Among the structural properties in complex systems, controllability and observability are still discussion topics for a wide variety of dynamical systems, from classic linear timeinvariant systems to other more complex families such as infinite-dimensional systems, stochastic systems, and hybrid systems, among others. This problem increases when they are subject to a large amount of connections, in which a classic handling is very difficult such as in complex networks [35], where the connection properties, such as symmetry $[36,37]$, and some computational tools have been developed to determine these properties [38].
In the case of stability of complex systems, two important aspects to consider are the time-delay between nodes (or the possible presence of time delay in the internal dynamics) and the robustness of the system (connection and internal) due to parametric variations. On the one hand, the time-delay induces infinite-dimensional dynamics whose equilibrium stability is still an active topic of research for, both, stability analysis itself and the use of the delay as a stabilizing element [39]. On the other hand, the parameter variations in a system demands a special stability treatment which has motivated some significant results such as Kharitonov's theorem [40] or $H_{\infty}$ loop shaping in control design [41].

In this article, a stability review for a class of systems subject to parameter variations and time delays is addressed. The review includes a definition of the dynamics of a family of nonlinear time-delay systems, a brief historical outlook of the stability study development, and then it gives some approaches in time and frequency domain, as well as a numerical analysis to provide the stability operating regions. Then, the robustness aspect of stabilizing controls is discussed on the realm of Hurwitz polynomials families. 
Finally, some perspectives of applications demanding these approaches are briefly provided.

\section{Stability and Stabilization of Linear Systems}

Characteristic root locations of linear systems or linear delayfree systems are completely related to system stability. The same criterion holds for linear time-delay system. Furthermore, many of the concepts and criteria for determining stability on time-delay system (TDS) are extensions or adaptations of the results initially proposed for linear delay-free systems. Accordingly, it is considered relevant to briefly address some classic stability criteria for this type of system.

In this section, some concepts and criteria regarding the stability of linear systems are analyzed. The section is divided as follows. In Section 2.1, the concept of stability in linear systems is addressed, Section 2.2 presents what can be the objectives in the stabilization of linear systems, and, finally, in Section 2.3, Kharitonov's theorem is analyzed and some families of polynomials to illustrate the importance of studying the stability in systems with uncertainties in the parameters are given.

2.1. Stability of Linear Systems. Lyapunov's doctoral thesis was perhaps the first systematic work on the subject of stability [42], although the study of stability theories was started by Maxwell, around 1868, in his work "on governors" [43]. Lyapunov presented a general definition of stability which referred to the stability of a solution of a differential equation, not necessarily at the equilibrium point.

Before Lyapunov, there were works that tried to explain the stability phenomenon in applications; it is worth mentioning the works of Lagrange and Dirichlet [44-48]. After Lyapunov's work, new concepts appeared such as uniform, global, exponential, and quasi asymptotic stability, among other types of them. In the following, some of the best known criteria for determining the stability of linear systems will be briefly mentioned.

A way to determine the asymptotic stability of continuous time-invariant systems is characterized by its eigenvalues. If all eigenvalues lie into open left half plane $C^{-}$, then the system is stable. A polynomial $p(s)$ that satisfies that all its roots are in $C^{-}$is called a Hurwitz polynomial or stable polynomial. Routh and Hurwitz showed, in independent works, that the stability of a matrix system could be determined by means of the coefficients of its characteristic polynomial. Their results are currently presented under the so-called Routh-Hurwitz criterion (see $[49,50])$ and can be considered as a numerical criterion. In view of that, it is reduced to the calculation of determinants. Another useful approach used to test the stability is the Hermite-Biehler criterion [51, 52], which can be considered as an algebraic criterion, which expresses the stability in terms of even and odds parts of the characteristic polynomial. A pair of polynomials $u$ and $v$ are said to be a couple positive if the principal coefficients of $u$ and $v$ have the same sign and the roots $\mu_{i}$ of $u$ and $v_{i}$ of $v$ alternate orderly on the real axis, i.e., are real, distinct, and negative and satisfy the interlacing property. So, the Hermite-Biehler theorem states that a polynomial $p(x)$ that can be written in the form $p(x)=$ $f\left(x^{2}\right)+x g\left(x^{2}\right)$ is stable if and only if $f$ and $g$ are a couple positive (see $[49,50]$, for details). There are others noncommon stability criteria; it is worth mentioning some of them. The Lienard-Chipart conditions [53] reduce the positivity evaluation of the main minors of the Routh-Hurwitz criterion to half.

The Leohnard-Mihailov criterion [54], which expresses the stability in geometric terms, analyzes the argument of the complex polynomial $p(i w)$ associated with the characteristic polynomial $p$ of the system. Routh's scheme gives rise to a recursive algorithm for testing the stability called Hurwitz stability test. Other approaches consider the Bezoutiant or the Cauchy indexes to verify stability of polynomials; for details, see $[49,50,55]$.

Not less important are those systems, where uncertainty is considered and incorporated through parameters. The robust stability of parameter uncertainty systems and the families of polynomials are associated with them, and it is addressed in the subsequent sections.

2.2. Stabilizing Controls. In this section, the relation between stabilizing feedback and some families of Hurwitz polynomials is explained. Consider the controllable system:

$$
\dot{x}(t)=A x(t)+b u(t)
$$

$x, b \in R^{n}$ and the controllable pair $(A, b)$ is given in the canonical form:

$$
\begin{aligned}
A & =\left(\begin{array}{ccccc}
0 & 1 & 0 & \cdots & 0 \\
0 & 0 & 1 & \cdots & 0 \\
\vdots & \vdots & \vdots & \cdots & \vdots \\
0 & 0 & 0 & \cdots & 1 \\
-a_{n} & -a_{n-1} & -a_{n-2} & \cdots & -a_{1}
\end{array}\right), \\
b & =\left(\begin{array}{c}
0 \\
0 \\
\vdots \\
0 \\
1
\end{array}\right) .
\end{aligned}
$$

Note that the open-loop polynomial is given by $p_{0}(s)=s^{n}+a_{1} s^{n-1}+\cdots+a_{n}$.

Now, let us define the feedback control $u$ as follows:

$$
u(t)=-k c^{T} x(t),
$$

where $c \in R^{n}$ and $k>0$. Then, the closed-loop polynomial is given by

$$
\begin{gathered}
p_{c}(s)=s^{n}+a_{1} s^{n-1}+a_{2} s^{n-2}+\cdots+a_{n}+k \\
\cdot\left(c_{1} s^{n-1}+c_{2} s^{n-2}+\cdots+c_{n}\right) .
\end{gathered}
$$

Let us denote $p_{c}^{*}(s)=c_{1} s^{n-1}+\cdots+c_{2} s^{n-2}+\cdots+c_{n}$. Then, $p_{c}(s)=p_{0}(s)+k p_{c}^{*}(s)$. Now, let us suppose that the 
$p_{c}^{*}(s)$ is a Hurwitz polynomial. When $k \longrightarrow \infty$ a closedloop eigenvalue, say $\lambda_{1}$ satisfies $\left(\lambda_{1} / k\right) \longrightarrow-c_{1}$, the other eigenvalues converge to the zeroes of $p^{*}(s)$, that is, when $k \gg 1$, feedback (3) is a stabilizing control (see $[56,57]$ ).

Also note that when $k \gg 1$, then high control gains $k c^{T}$ are induced in the feedback (3); hence, feedback (3) is a highgain feedback. There are several studies to analyze the properties of high-gain controls (see [56-64]).

Returning to the analysis of the closed-loop polynomial $p_{c}(s)=p_{0}(s)+k p_{c}^{*}(s)$, it can be seen that $p_{c}(s)$ is not necessarily Hurwitz for all $k>0$, even when $p_{0}(s)$ is Hurwitz and $c \in R^{n}$ is chosen such that $p_{c}^{*}(s)$ is a Hurwitz polynomial (see [65-67]).

The last observation is illustrated with the following example, which was presented in [67].

Consider the system:

$$
\dot{x}(t)=\left(\begin{array}{ccc}
0 & 1 & 0 \\
0 & 0 & 1 \\
-5 & -11 & -7
\end{array}\right) x(t)+\left(\begin{array}{l}
0 \\
0 \\
1
\end{array}\right)+(-65 k,-3 k,-k) x(t) .
$$

Here, $\quad p_{0}(s)=s^{3}+7 s^{2}+11 s+5=(s+1)^{2}(s+5) \quad$ and $p_{c}^{*}(s)=s^{2}+3 s+65, p_{0}(s)$ and $p_{c}^{*}(s)$ are Hurwitz polynomials. However, $p_{0}(s)+k p_{c}^{*}(s)$ is not a Hurwitz polynomial for $k \in[3,8]$; hence, the importance of have methods for choosing vectors $c$ such that $u(t)=-k c^{T} x(t)$ is a stabilizing control. Consequently, it is necessary to study the Hurwitz stability of the ray of polynomials $p_{0}(s)+k p_{c}^{*}(s)$ with $k>0$.

In the next section, a technique in terms of rays and segments of polynomials is presented.

2.2.1. Relation between Stabilizing Controls and Rays and Segments of Hurwitz Polynomials. As can be observed above, the control $u(t)=-k c^{T} x(t)$ is a stabilizing feedback if and only if the ray of polynomials $p_{0}(s)+k p_{c}^{*}(s)$ is a ray of Hurwitz polynomials. On the contrary, there is an obvious relation between Hurwitz rays and Hurwitz segments of polynomials: if $p_{0}(s)+k p_{c}^{*}(s)$ is a Hurwitz polynomial, then $(1 / 1+k) p_{0}(s)+(k / 1+k) p_{c}^{*}(s)$ is a Hurwitz polynomial, which implies that the Hurwitz stability of the ray $p_{0}(s)+k p_{c}^{*}(s)$ is equivalent to the Hurwitz stability of the segment of polynomials $\left[p_{0}(s), p_{c}^{*}(s)\right]$.

The problem to establish conditions on the Hurwitz polynomials $f_{0}(s)$ and $f_{1}(s)$ such that the segment of polynomials determined by $f(s, \lambda)=\lambda f_{0}(s)+(1-\lambda) f_{1}(s)$ is Hurwitz stable for all $\lambda \in[0,1]$ has been studied with different approaches (see [65-73]). The first reported work about this subject is Bialas's paper [68]. Bialas's theorem says that if $f_{0}(s)$ is a Hurwitz polynomial and $\operatorname{deg}\left(f_{0}\right)>\operatorname{deg}\left(f_{1}\right)$, then $f(s, \lambda)$ is Hurwitz for all $\lambda \in[0,1]$ if and only if the matrix $H^{-1}\left(f_{0}\right) H\left(f_{1}\right)$ has no eigenvalues in $(-\infty, 0)$, where $H(p)$ is the Hurwitz matrix of the polynomial $p$ (see $[68,74,75])$.

Other method which is known as the segment Lemma was obtained by Chapellat and Bhattacharyya (see $[76,77]$ ). The segment lemma is an approach that presents conditions in the frequency domain. Based on the segment lemma, a computational algorithm was developed in [71] for testing the Hurwitz stability of segments of polynomials. On the contrary, Bose developed a technique to check the stability of segments of complex polynomials [78].

Based on Bose's test, in [69], a test for checking the stability of segment of complex polynomials was obtained. Another computational method is presented in [79]. Sufficient conditions to guarantee the Hurwitz stability of segments were obtained by Rantzer (see $[72,80])$. On the contrary, an approach, where sufficient conditions in terms of matrix inequalities for checking the Hurwitz stability of segments of polynomials, has been presented in [65-67, 73]. The explanation of the aforementioned approach is the following: let $f_{0}(s)=s^{n}+a_{1} s^{n-1}+\cdots+a_{n}$ be a Hurwitz polynomial.

Consider the matrix $E_{(n, n-1)} \in M_{n \times n}$ defined by

$$
E_{(n, n-1)}=\left(\begin{array}{ccccccc}
a_{1} & -1 & 0 & 0 & \cdots & 0 & 0 \\
-a_{3} & a_{2} & -a_{1} & 1 & \cdots & 0 & 0 \\
a_{5} & -a_{4} & a_{3} & -a_{2} & \cdots & 0 & 0 \\
\vdots & \vdots & \vdots & \vdots & \cdots & \vdots & \vdots \\
0 & 0 & 0 & 0 & \cdots & a_{n-1} & -a_{n-2} \\
0 & 0 & 0 & 0 & \cdots & 0 & a_{n}
\end{array}\right),
$$

and consider the matrix $D_{(n, n-1)} \in M_{n \times n}$ defined by

$$
D_{(n, n-1)}=\left(\begin{array}{ccccccc}
1 & 0 & 0 & 0 & \cdots & 0 & 0 \\
-a_{2} & a_{1} & -1 & 0 & \cdots & 0 & 0 \\
a_{4} & -a_{3} & a_{2} & -a_{1} & \cdots & 0 & 0 \\
\vdots & \vdots & \vdots & \vdots & \cdots & \vdots & \vdots \\
0 & 0 & 0 & 0 & \cdots & a_{n-2} & -a_{n-3} \\
0 & 0 & 0 & 0 & \cdots & -a_{n} & a_{n-1}
\end{array}\right) .
$$

Now, let $f_{1}(s)=c_{1} s^{n-1}+c_{2} s^{n-2}+\cdots+c_{n}$ be an arbitrary polynomial of degree $n-1$ with positive coefficients. If the vector $\left(c_{1}, c_{2}, \ldots, c_{n}\right)^{T}$ satisfies the system of linear inequalities $E_{(n, n-1)} c \supsetneqq 0$ or $D_{(n, n-1)} c \supsetneqq 0$, then $\lambda f_{0}(s)+(1-$ d) $f_{1}(s)$ is Hurwitz for every $\lambda \in[0,1]$ (here the symbol $>0$ $(<0)$ means that the components of a vector are nonnegative (nonpositive) and the symbol $\gtreqless$ means that all of the components of a vector are nonnegative, but there is at least one positive component).

Other interesting references about segments of Hurwitz polynomials are the works [74, 76, 81-86]. Besides, in relation with Hurwitz polynomials, it is worth to consulting paper [87].

2.3. Robust Stability. The presence of several uncertain parameters in description of a LTI system manifests itself as variations in the coefficients of the characteristic polynomial. The determination of stability and stability margins under parametric uncertainty, structure uncertainty itself included, is the main purpose of the robust stability. 
Perhaps, the most famous result about the Hurwitz polynomial families is the Kharitonov theorem, which is related to the interval-type polynomials. This section presents this theorem and addresses some related results.

2.3.1. The Kharitonov Theorem. The problem of stability under large parameter uncertainty was strongly promoted with the advent of a remarkable theorem due to the Russian control theorist V. L. Kharitonov.

Consider the interval family of polynomial defined by

$$
P(s)=\alpha_{0}+\alpha_{1} s+\alpha_{2} s^{2}+\cdots+\alpha_{n} s^{n},
$$

where

$$
\alpha_{0} \in\left[a_{0}, b_{0}\right], \alpha_{1} \in\left[a_{1}, b_{1}\right], \ldots, \alpha_{n} \in\left[a_{n}, b_{n}\right] .
$$

Consider the following four elements of the family, named the Kharitonov polynomials:

$$
\begin{aligned}
& k^{1}(s)=\left(a_{0}+a_{1} s+b_{2} s^{2}+b_{3} s^{3}+a_{4} s^{4}+a_{5} s^{5}+\ldots\right), \\
& k^{2}(s)=\left(a_{0}+b_{1} s+b_{2} s^{2}+a_{3} s^{3}+a_{4} s^{4}+b_{5} s^{5}+\ldots\right), \\
& k^{3}(s)=\left(b_{0}+a_{1} s+a_{2} s^{2}+b_{3} s^{3}+b_{4} s^{4}+a_{5} s^{5}+\ldots\right), \\
& k^{4}(s)=\left(b_{0}+b_{1} s+a_{2} s^{2}+a_{3} s^{3}+b_{4} s^{4}+b_{5} s^{5}+\ldots\right) .
\end{aligned}
$$

Kharitonov's theorem establishes that every polynomial in families (8) and (9) is Hurwitz if and only if the four Kharitonov polynomials $10(10)$ are Hurwitz. This result has been the motivation of different extensions, alternate proofs, and applications to some classes of families of polynomials, for instance,

(i) The problem to find conditions for family (8) to be Hurwitz was planted by Faedo [88]

(ii) The original proof was given by Kharitonov in [89], but different authors have presented other proofs (see [70, 77, 90-93])

(iii) Kharitonov extended his result to the complex case in 1979 [94]

(iv) Generalizations of Kharitonov's theorem are presented in $[56,95,96]$

(v) Applications of Kharitonov's theorem can be consulted in [97]

(vi) Recent information about Interval Families and Kharitonov's theorem was published in papers $[65,98,99]$

The appearance of Kharitonov's theorem led to a resurgence of interest in the study of robust stability under real parametric uncertainty. In Section 2.2, some results related to stability of certain families of polynomials such as the segments and the rays of polynomials were mentioned. Some other families worth mentioning are the ball of stable polynomials and polytope of polynomials. The ball of stable polynomials is a way of characterizing the largest region where the stability of a family of polynomials is preserved. Soh et al. [100] in 1985 adopted a point of view opposite to Kharitonov. Starting with an already stable polynomial $p(s)$, they gave a way to compute the radius of the largest stability ball in the space of polynomial coefficients around $p$. The estimation for the $l_{2}$-norm stability ball in the space of coefficients was calculated by Soh et al. For $l_{p}$-norm, the calculation was realized by Tsypkin and Polyak [101].

Otherwise, the main robust stability result related with polytope of polynomials is the celebrated Edge theorem of Bartlett et al. [102], which considers more general stability regions, and it is not restricted to Hurwitz stability. They considered a family of polynomials whose coefficients vary in an arbitrary polytope: $p(s)=a_{1} Q_{1}(s)+a_{2} Q_{2}(s)+\cdots+$ $a_{m} Q_{m}(s)$ on $R^{n+1}$, with its edges not necessarily parallel to the coordinate axes as in Kharitonov's problem. They proved that the root space of the entire family is bounded by the root loci of the exposed edges. In particular, the entire family is stable in and only if all the edges are proved to be stable. The key idea behind this result is that we can reduce a multidimensional uncertainty problem into a finite number of one-parameter problems whose solution requires less effort.

One of the most used tools in the analysis of robust stability in families of polynomials, where the coefficients depend continuously on a set of parameters, is the Boundary Crossing theorem and its computational version the Zero Exclusion principle. Consider a family of polynomials $F(s, p)$ of degree $n$, where the real parameter $p$ ranges over a connected set $\Omega$. If it is known that one member of the family is stable, a useful technique of verifying robust stability of the family is to ascertain that $F(j \omega, p) \neq 0$ for all $p \in \Omega, \omega \in R$. This can also be written as the zero exclusion condition $0 \notin F(j \omega, \Omega)$, for all $\omega \in R$. This zero exclusion condition has been exploited to derive various types of robust stability and performance margins.

\section{Stability and Stabilization of Time-Delay Systems}

This section begins with a brief classification of nonlinear TDS, followed by a recurring classification in which the nonlinear TDS has a nominal part (linear part). The above allows to show basic and pillar results existing in the literature for the stability and stabilization analysis of linear TDS in two domains, frequency and temporal. Although it is typically believed that an analysis in the frequency domain is only limited to linear systems, recent results have shown that a study on polytope of quasi-polynomials (generalized characteristic quasi-polynomial) can determine stability conditions for a class of nonlinear TDS. Furthermore, in the time domain, the use of complete type Lyapunov-Krasovskii functionals can provide necessary and sufficient stability conditions for a class of linear TDS, while reduced type functionals only give sufficient conditions.

The understanding, analysis, and prediction of the dynamics of a system are topics that generate considerable interest in the scientific community. When this system presents nonlinearities and delays in its structure, the useful information is even richer, since it is more consistent to the dynamics observed in the systems/processes/prototypes of the physical world. These types of systems are known as nonlinear time-delay systems or nonlinear systems with 
delays. Sometimes, nonlinear dynamics are phenomena that often introduce unpredictable chaotic behaviors into a system, whereas the delays are due to the fact that the rate of variation in the system dynamics depends on past states, which implies an analysis in an infinite-dimensional space, and this is in mathematical terms. Thus, the dynamics observed in communication networks [103-105], teleoperation [106, 107], chemical processes [108], population dynamics [109], biological phenomena [110], game theory and economic applications [111, 112], unmanned aerial vehicles [113], haptic interfaces [114], and robotic systems $[115,116]$, among others, can be mathematically modeled using nonlinear time-delay systems.

The nonlinear TDS are usually represented by functional differential equations (FDEs) also known as delay differential equations. Among functional differential equations, one may distinguish some particular classes as retarded functional differential equations (RFDEs) (or functional differential equations of retarded type), neutral functional differential equations (NFDEs) (or functional differential equations of neutral type), distributed functional differential equations (DFDEs) (or functional differential equations of distributed type), and differential-difference equations (DDEs). For illustrative purposes and to characterize the research space of the FDEs, the form of RFDE with one delay is presented below:

$$
\begin{aligned}
\dot{x}(t) & =f\left(t, x_{t}\right), \\
x(\theta) & =\phi(\theta), \quad \theta \in[-\tau, 0],
\end{aligned}
$$

where $f: R^{+} \times \mathfrak{C} \longrightarrow R^{n}$ is continuous and satisfies a local Lipschitz condition regarding the second element of the argument. For $t \geq 0$, denote by $x(t, \phi) \in R^{n}$ the system solution with initial function (condition) $\phi \in \mathfrak{C}$, and by $\mathfrak{V}:=C\left([-\tau, 0], R^{n}\right)$ the Banach space with norm $\|\phi\|_{\tau}:=\max _{\theta \in[-\tau, 0]}\|\phi(\theta)\|$. Here, $\|\cdot\|$ denotes the Euclidean norm. As a natural extension of the initial function, a solution segment of $x(t, \phi)$ in a time interval $[t-\tau, t]$ is denoted by

$$
x_{t}=x(t+\theta) \in \mathfrak{C}, \quad \theta \in[-\tau, 0],
$$

and called state of system (11). In turn, the above RFDE can be classified as follows:

(i) Time-invariant RFDE if the first term of the argument is omitted:

$$
\begin{aligned}
\dot{x}(t) & =f\left(x_{t}\right), \\
\text { or } \dot{x}(t) & =f(x(t), x(t-\tau)) .
\end{aligned}
$$

(ii) RFDE with multiple delay if $0=\tau_{0}<\tau_{1}<\cdots<\tau_{m}=$, that is,

$$
\dot{x}(t)=f\left(t, x(t), x\left(t-\tau_{1}\right), x\left(t-\tau_{2}\right), \ldots, x\left(t-\tau_{m}\right)\right) .
$$

(iii) State-dependent delay RFDE if $\tau$ depends on state, $x_{\tau}$ :

$$
\dot{x}(t)=f(t, x(t), x(t-\tau(x(t)))) .
$$

(iv) Time-varying delay RFDE if the delay $\tau$ depends on $t$ :

$$
\dot{x}(t)=f(t, x(t), x(t-\tau(t))) .
$$

(v) RFDE with distributive delay, also known as DFDE, if the delay is represented as a continuously distribution and it is not instantaneous:

$$
\dot{x}(t)=f\left(t, x(t), \int_{0}^{\infty} x(t-\vartheta) d \vartheta\right) .
$$

(vi) RFDE with neutral delay or NFDE if the system also depends on the time derivative of the state:

$$
\begin{aligned}
\dot{x}(t) & =f\left(t, x_{t}, \dot{x}_{t}\right), \\
\text { or }\left(\frac{\mathrm{d}}{\mathrm{d} t}\right)\left[x(t)-g\left(t, x_{t}\right)\right] & =f\left(t, x_{t}\right) .
\end{aligned}
$$

(vii) RFDE with discrete delay or DDE if $\tau$ is the interval between the successive sample instants $t_{k-1}$ and $t_{k}$, $\tau=t_{k+1}-t_{k}:=\Delta t, k=1,2, \ldots, N$ and $t=(N-1)$. Here, $\tau$ is called the sample period, and $x_{k}=x(t-$ $(N-k) \tau)=x(t-(N-k) \Delta t)$ is an $N$-dimensional discrete mapping, and using any integral scheme we obtain

$$
\begin{aligned}
x(t+\Delta t) & =x(t)+f\left(x, x_{\tau}\right) \Delta t, \quad x_{\tau}=x(t-\tau) \text { or, } \\
x_{1}(k+1) & =x_{N}(k)+f\left(x_{N}(k), x_{1}(k)\right) \Delta t, \\
x_{2}(k+1) & =x_{1}(k+1)+f\left(x_{1}(k+1), x_{2}(k)\right) \Delta t, \\
\vdots & \\
x_{N}(k+1) & =x_{N-1}(k+1)+f\left(x_{N-1}(k+1), x_{N}(k)\right) \Delta t .
\end{aligned}
$$

The above, as well as the possible combinations between these, are just some types of TDS. Currently, there is a broader classification of systems that are outside this basic classification. In addition, each type of TDS needs more appropriate concepts and descriptions for the research space and existence and uniqueness of the solution, among others. For further information, the reader is referred to the following authoritative references [52, 117-127]. Although these themes are very interesting, the focus of this review is on stability and stabilization. Therefore, it will be the next topic to discuss.

3.1. Stability of Time-Delay Systems. Undoubtedly, one of the most important research topics for the TDS research community is the analysis of stability. In this context, obtaining sufficient and/or necessary conditions to determine when the studied system remains stable or when it gains and/or loses stability is an important topic for the community. Furthermore, the knowledge of these conditions allows to solve other problems associated with this 
topic such as analysis of robustness/adaptability/uncertainty/perturbation, design of observers, synthesis and tuning of controllers, determination of attraction regions estimates, and study of chaotic/hyperchaotic behaviors, to name just a few, [128-137].

Typically, the stability of the TDS is studied on two main frameworks: frequency domain and time domain. The fundamental results were proposed by Pontryagin $[138,139]$, Wright $[140,141]$, Bellman [142, 143], and Cooke [144] in the 1940s and 1950s. These results are in the frequency domain, which is based on a study of its corresponding characteristic equation (exponential polynomial, quasi-polynomial, and analytic function with transcendental terms) to determine the location of its roots in the complex plane or from the nontrivial solutions of a delayed Lyapunov matrix function. Later, Razumikhin [145] and Krasovskii [146] proposed to extend Lyapunov's results to analyze stability of TDS in the time domain.

The stability of nonlinear TDS has been studied for almost 80 years and most of the results proposed by the research community are about nonlinear systems with a specific structure. Among which, it is possible to apply techniques that benefit/facilitate the analysis of the stability of complex systems around an operating (equilibrium) point or even in a sector of it. Since, these allow to rewrite exactly or approximately a complex system in a more accessible system to study. Techniques such as sector nonlinearity [147], tangent linearization [148], feedback linearization approach (Lie derivative) [149, 150], passification [151-154], backstepping [155-158], immersion and invariance [159-162], and differential flatness [163-165], among others. On the contrary, in some cases, a linearization is proposed around an operating point. While in other cases, the nonlinear systems is represented with a dominant part (dominant linear part) plus nonlinear part is proposed. In this way, in the nonlinear part, disturbances, nonmodel dynamics, and parametric variations, among others, are usually introduced. Below, it is a classification of the aforementioned systems.

Let a nonlinear TDS be given in (11); in some cases, this system can be represented as

$$
\dot{x}(t)=L\left(t, x_{\tau}\right)+g\left(t, x_{t}\right),
$$

where $L: R^{+} \times \mathfrak{C} \longrightarrow R^{n}$ is a linear operator and $g: R^{+} \times$ $\mathfrak{S} \longrightarrow R^{n}$ is a nonlinear operator, both properly defined. Typically, if $g$ satisfies certain conditions (bounded, Lipschitz, and quasi-Lipschitz, among others), then $g$ is considered as an uncertainty or/and perturbation of the nonlinear TDS, so the stability analysis of (20) focuses on the nominal system $\dot{x}(t)=L\left(t, x_{\tau}\right)$. A classification of the nominal part $L\left(t, x_{\tau}\right)$ proposed here is as follows:

(i) $L\left(t, x_{\tau}\right)=A_{0}(t) x(t)+A_{1}(t) x(t-\tau)$ is a linear time-variant (LTV) delay system of retarded type

(ii) $L\left(t, x_{\tau}\right)=A_{0}(\theta(t)) x(t)+A_{1}(\theta(t)) x(t-\tau)$ is a linear parameter varying (LPV) system with time delay, where $\theta$ are uncertain time-variant real parameters which satisfy $\underline{\theta} \leq \theta(t) \leq \bar{\theta}$ (iii) $L\left(t, x_{\tau}\right)=A_{0} x(t)+A_{1} x(t-\tau(t))$ is a linear system with time-varying delay, where $0 \leq \tau(t) \leq \bar{\tau}$ and $\dot{\tau}(t) \leq \mu \leq$, for all $t \geq 0$

(iv) $L\left(t, x_{\tau}\right)=A_{0} x(t)+A_{1} x(t-\tau)$ is a linear time-invariant (LTI) TDS of retarded type

(v) $L\left(t, x_{\tau}\right)=A_{0} x(t)+A_{1} x(t-\tau)+D_{0} \dot{x}(t)+D_{1} \dot{x}(t-\tau)$ is a linear NFDE (linear neutral time-delay systems or linear time-delay system of type neutral), where $\left|D_{i}\right|<1$, for any matrix norm $|\cdot|$

(vi) $L\left(t, x_{\tau}\right)=A_{0} x(t)+A_{1} x(t-\tau)+\int_{\tau}^{0} D(\vartheta) x(t+\vartheta) d$ is a linear DFDE or linear distributed time-delay system; here, $D(t)$ is a continuous matrix on $[-\tau, 0]$ of appropriate dimensions

(vii) $L\left(t, x_{\tau}\right)=\sum_{i=0}^{m} A_{i} x\left(t-\tau_{i}\right)$ is a LTI-TDS with multiple delays of retarded type, $0=\tau_{0}<\tau_{1}<\cdots<\tau_{m}=\tau$

To learn more about the systems described above, the reader can consult the following references [52, 166-169]. Although the nonlinear part $g\left(t, x_{\tau}\right)$ is also important and its structure depends on the type of studied nonlinear system, it is more important to know the studies regarding the stability of the nominal part. Since the stability of the nominal part can contribute the obtaining of robust stability conditions in the presence of the nonlinear part. Therefore, some research studies on stability analysis of linear TDS in the frequency domain and time domain are presented below.

3.2. Stability in the Frequency Domain. It is well known that the analysis of stability and stabilization of a TDS in the frequency domain is based on a study of its corresponding characteristic equation to determine the location of its roots in the complex plane. This concept is inherited from the stability analysis of delay-free systems, mainly from Hurwitz's concept $[170,171]$. However, when the delays are considered in a system, it involves the inclusion of transcendental terms in the characteristic equation, changing the analysis of a polynomial (free-delay systems) to a quasipolynomial (TDS). This complicates the analysis of a finite number to an infinite number of roots [172], also this analysis is usually limited to LTI-TDS. However, in contrast with the time domain, this analysis allows obtaining necessary and sufficient stability conditions; recent results have shown that an analysis in the frequency domain can be applied to a class of nonlinear TDS, see [173, 174].

Given a LTI-TDS with multiple delays of retarded type,

$$
\dot{x}(t)=\sum_{i=0}^{m} A_{i} x\left(t-\tau_{i}\right)
$$

then its quasi-polynomial is of the form

$$
\begin{aligned}
Q(s) & =\operatorname{det}\left\{s I_{n}-\sum_{k=0}^{m} A_{k} e^{-s \tau_{k}}\right\} \\
& =\sum_{k=0}^{m} p_{k}(s) e^{-s \tau_{k}}=0
\end{aligned}
$$


where $I_{n}$ is the identity matrix of $n$-dimension, $0=\tau_{0}<$ $\tau_{1}<\cdots<\tau_{m}=\tau, \quad p_{k}(s)=\sum_{j=0}^{r_{k}} a_{k} s^{j}, \quad k=0,1, \ldots, m$, are polynomials with real coefficients and $\operatorname{deg}\left(p_{0}\right) \leq \operatorname{deg}\left(p_{k}\right)$, i.e., $r_{0} \leq r_{k}, k=1, \ldots, m$. Although $Q(s)$ given in 3.2 has an infinite number of roots, it is enough to know the location of the dominant roots as shown below.

Definition 1 (see [166]). The LTI-TDS with multiple delays of retarded type (21) is said to be $\sigma$-stable (exponentially stable) if the system response $x(t, \phi)$ satisfies the following inequality:

$$
\|x(t, \phi)\| \leq L e^{-\sigma t}\|\phi\|_{\tau}, \quad t \geq 0
$$

where $L>0, \sigma \geq 0$, and $\phi:[-\tau, 0] \longrightarrow C\left([-\tau, 0], R^{n}\right)$ is the initial condition.

Under consideration, for LTI-TDS of retarded type, the exponential stability and asymptotic stability are equivalent.

Definition 2 (see [166]). Consider the quasi-polynomial 3.2, $\sigma \in R$ a positive constant and

$$
s_{0}=\max _{j=1, \ldots, \infty}\left\{\operatorname{Re}\left\{s_{j}\right\} \mid Q\left(s_{j}\right)=0, s_{j} \in C\right\},
$$

where $\operatorname{Re}\left\{s_{j}\right\}$ denotes the real part of $s_{j}$. Then, the LTI-TDS with multiple delays of retarded type (22) is $\sigma$-stable if $s_{0} \leq-\sigma$ (relative stability, [175]).

As can be seen in the above definitions, the stability of an LTI-TDS with multiple delays depends on the dominant roots of 3.2, which determine the abscissa $-\sigma$ (spectral abscissa) or vertical line on $-\sigma$ in the complex plane. Furthermore, the roots have continuous variations with respect to parametric variations of the system. This is known as continuity property.

Theorem 1 (see [176]). If the matrices $A_{k}$ or the delays $\tau_{k}$, $k=1,2, \ldots, m$, are varied, then a loss or acquisition of exponential stability of the solution $x(t, \phi)$ of LTI-TDS with multiple delays (21) is associated with the dominant roots of the quasi-polynomial (22).

This allows to obtain conditions of robustness when there are parametric variations and also the design and tuning of control laws for $s$-stabilize TDS are as shown in Section 3.4.

However, in the framework of TDS stability in the frequency domain, there are many results/criteria; among the first and most important are the following:

(i) The Pontryagin criterion [177] is considered as one of the most general analytical criteria, and it gives necessary and sufficient conditions for the stability of (22). However, it has strong limitations and may become very complicated for systems with more than one delay.

(ii) The Yesipovich-Svirskii criterion [178] is for systems with one discrete delay. The necessary and sufficient condition of the stability of (22) is given by means of the expression:

$$
\sum_{k} \operatorname{sgn}\left(\left(\frac{1}{\operatorname{Re}\left\{F\left(u_{k}\right)\right\}}\right)\left(\frac{\operatorname{dIm}\left\{F\left(u_{k}\right)\right\}}{d\left(u_{k}\right)}\right)\right),
$$

where

$$
F(z)=\frac{1}{\tan (1 / 2 z)}+\phi(z)
$$

is a further transformation $s=i z, i^{2}=-1, z \in C$, of $Q(s)=0, \phi(z)$ is a function that does not contain transcendental terms, and $u_{k}$ are the real roots of $\operatorname{Im} \phi(z)=0$.

(iii) The $\tau$-decomposition method [179] requires the transformation of the quasi-polynomial into the form

$$
e^{\tau s}=D_{0}(\tau)
$$

where $D_{0}(s)$ is a ration of two polynomials. This method is for systems with one discrete delay, and it is based on the analysis of the contour $D_{0}(i u)$, $u \in R^{+}$around the unit circle in the complex plane.

(iv) The principle of argument [121] is used to determine the number $N$ of roots of $Q(s)$ inside of an closed curve $C \subset C$, where

$$
N=\left(\frac{1}{2 \pi}\right) \Delta_{C \arg }\{Q(s)\}
$$

Here, $\Delta_{C} \arg \{Q(s)\}$ denotes the changes of the argument of $Q$ along $C$.

(v) The Chebotarev criterion is the direct generalization of the Routh-Hurwitz. The analytical criterion needs to calculate an infinite number of Hurwitz determinants and the stability of the system with long delay is determined by determinants of high dimension, whereby it is not effective practically.

(vi) The D-partition (D-subdivision) method [180] is a geometric method to construct stability charts (regions) in the parameter space of the quasipolynomial. It is very effective to determine stability based on system parameters. However, the number of parameters used is reduced.

(vii) The Nyquist [181] method is also a geometric method and the stability of the systems is determined by the relative position of the point $-1+0 i$ and the contour $D(i u) \subset C, i^{2}=-1, u \in R^{+}$, where $-1=D(s)$ is a transformation of the quasi-polynomial.

(viii) The Bode and Nichols criteria and some others are transformations of the Nyquist criterion as Satche mentions in [182].

(ix) The Mikhailov criterion [183] is a consequence of the Cauchy Residue theorem in complex analysis. It can be applied to RFDE if there exists a scalar $\nu>0$ such that the quasi-polynomial is bounded and analytical in any closed domain in $\{s: \operatorname{Re}\{s\}>v\}$; this is also known as Satche's diagram [184]. 
(x) The Hermite-Biehler criterion [51, 52] mentioned in the previous section can be applied for TDS using the imaginary and real parts of the quasi-polynomial, $Q(s=i \omega)=\operatorname{Re}\{Q(i \omega)\}+\operatorname{Im}\{Q(i \omega)\}, \quad i=$ $Q_{r}(\omega)+Q_{i}(\omega) i$; the stability of the system is determined by a continuous alternation between transformations of the real functions $Q_{r}(\omega)$ and $Q_{i}(\omega)$, when increasing phase condition $\omega>\omega^{*}$, for any $\omega^{*} \in(-\infty, \infty)$. See its extensions in $[185,186]$.

(xi) The Edge theorem [187], zero exclusion principle, and concept of convex direction [188] are graphical methods to determine stability of a set of quasipolynomial family or convex polytope family.

The above criteria are the most recurrent on stability analysis of quasi-polynomials, see [176, 189-194], but there are quasi-polynomial classes of larger complexity that require special attention. Among this class, the quasi-polynomial of NFDE [195], polynomial family which is described by convex polytope in the coefficient space [196], and stability analysis of LPV-TDS through a generalized characteristic quasi-polynomial [173] can be found.

Consider a LTI-NFDE with multiple delays of the form:

$$
\sum_{k=0}^{m} D_{k} \dot{x}\left(t-\tau_{k}\right)=\sum_{k=0}^{m} A_{k} x\left(t-\tau_{k}\right),
$$

where $A_{k}, D_{k} \in R^{n \times n}, k=0,1, \ldots, m$ and $0=\tau_{0}<\tau_{1}<\ldots$ $<\tau_{m}=\tau$. The corresponding quasi-polynomial is given by

$$
Q(s)=\operatorname{det}\left\{\left\{s I_{n}-\sum_{k=0}^{m} D_{k}\right\}-\sum_{k=0}^{m} A_{k}\right\}=0 .
$$

A TDS has an infinite number of characteristic roots, but $Q(s)$ is an entire function, which implies that there can only be a finite number of characteristic roots within any bounded domain. These characteristic roots form root chains that are rather easy to describe. The quasi-polynomial has two types of root chains. The first type is retarded chains; here, the roots fall in the region $|\operatorname{Re}\{s+\mu \log s\}|<c$, for some $\mu>0$ and $c>0$. In other words, there may only be a finite number of roots on the right of the abscissa $\alpha=\operatorname{Re}\{s\}$ in the complex plane for any given $\alpha$ [195]. The second type is neutral chains; here, the roots are bounded by two abscissas $c_{1} \leq \operatorname{Re}\{s\} \leq c_{2}$. The positions of such abscissas are determined by

$$
\operatorname{det}\left\{\left(I_{n}-\sum_{k=0}^{m} D_{k}\right)\right\}=0
$$

Additionally, if $s^{*}$ is a solution of (31); then, there is a series of roots $s_{l}, l=1,2 \ldots$ of quasi-polynomial (30) such that $\left|s_{l}\right| \longrightarrow \infty$ and $\operatorname{Re}\left\{s_{l}\right\} \longrightarrow \operatorname{Re}\left\{s^{*}\right\}$. Due to all the above, the stability of (30) is associated with the stability of $\mathrm{fd} 38(31)$. Furthermore, (31) can be sensitive to infinitesimal delay perturbations, which strongly affect the continuity property of the roots of (30). Moreover, in contrast with the retarded case, this property cannot be ensured with respect to parametric variations of the system. For more details on this type of systems, see [176, 195].
Another interesting study in the TDS research community is the stability analysis of convex sum of quasi-polynomials, known as polynomial family or convex polytope or polytope of quasi-polynomials. These are quasi-polynomials that are entire functions which include both degree of the independent variable and exponential functions and they appear when several subsystems with delays are interconnected. Consider a convex hull of quasi-polynomials of the form:

$$
\mathbf{Q}=\left\{\mathrm{Q}(s)=\sum_{r=1}^{M} \mu_{r} Q_{r}(s): \sum_{r=1}^{M} \mu_{r}=1, \mu_{r} \geq 0, r=1,2, \ldots, M\right\},
$$

where the vertex quasi-polynomial $Q_{r}(s)$ is of form (22). The stability of this class of the quasi-polynomials of family $\mathbf{Q}$ is studied using the zero exclusion principle, the concepts of convex direction, and the Edge theorem [196-199]. Here, a coefficient vector $a=\left(a_{00}, \ldots, a_{0 m}, a_{10}, \ldots, a_{1 m}, \ldots, a_{r_{k} 0}\right.$, $\left.\ldots, a_{r_{k} m}\right)$ is associated with every element $Q(s) \in Q$; then, family (32) can be described by the convex polytope:

$$
M_{\mathbf{Q}}=\left\{a=\sum_{r=1}^{M} \mu_{r} a_{r}: \sum_{r=1}^{M} \mu_{r}=1, \mu_{r} \geq 0, r=1,2, \ldots, M\right\},
$$

where the vector $a_{r}$ corresponds to the vertex quasi-polynomial $Q_{r}(s)$. Thus, $E\left(M_{\mathbf{Q}}\right)$ is the set of edges and $V\left(M_{\mathbf{Q}}\right)$ is the set of vertexes of the polytope $M_{\mathrm{Q}}$. Every edge corresponds to the one-parameter family of quasi-polynomial of the form $\mu Q_{p}(s)+(1-\mu) Q_{q}(s), \mu \in[0,1]$, i.e., the stability analysis for the families $\mathbf{Q}$ is reduced to a finite number of simpler problem stability to convex couples.

Theorem 2 (see [197]). The family $\mathbf{Q}$ is stable if and only if all members of one-parameter family corresponding to the edges $E\left(M_{\mathbf{Q}}\right)$ are stable.

On stability analysis of LPV-TDS, it seems that this topic is one of the most relevant topics and the best opportunity field to direct the current research. An aircraft is one classic physical system where the mathematical model can be represented by a LPV system [200], while a system of distributed type can be seen in [201] and a LPV-TDS in [173].

Consider a LPV-TDS of the form:

$$
\dot{x}(t)=\left(\begin{array}{cc}
0 & 1 \\
\theta(t)-2 & 0.1
\end{array}\right) x(t)+\left(\begin{array}{cc}
0 & 0 \\
-\theta(t)+1 & 0
\end{array}\right) x(t-\tau),
$$

where $\theta(t) \in[-1,1]$. Now, consider the LTV-TDS of the form:

$$
\begin{aligned}
\dot{x}(t)= & \left(\begin{array}{cc}
0 & 1 \\
\sin (t)-2 & 0.1
\end{array}\right) x(t) \\
& +\left(\begin{array}{cc}
0 & 0 \\
-\cos (t)+1 & 0
\end{array}\right) x(t-\tau),
\end{aligned}
$$

and the nonlinear TDS as follows: 


$$
\dot{x}(t)=\left(\begin{array}{cc}
0 & 1 \\
\sin \left(x_{1}\right)-2 & 0.1
\end{array}\right) x(t)+\left(\begin{array}{cc}
0 & 0 \\
-\cos \left(x_{2}\right)+1 & 0
\end{array}\right) x(t-\tau) .
$$

In terms of stability, the previous three systems (34)-(36) can have the same properties. In other words, the convex representation of the uncertainty of $\theta(t)$ and the nonlinear functions $(\sin (t), \cos (t))$ and $\left(\sin \left(x_{1}\right), \cos \left(x_{2}\right)\right)$ are equivalent in a stability analysis if only the manipulation range of the previous variables is considered. This undoubtedly provides the opportunity to obtain stable conditions for a wide variety of types of systems. Furthermore, these conditions can be obtained using LMIs and by studying a generalized characteristic quasi-polynomial of the form

$$
Q(s)=e^{s \tau_{m}} \operatorname{det}\left\{s I_{n}-A_{1}(\theta)-A_{1}(\theta) e^{-s \tau}\right\}=\sum_{k=0}^{n} \sum_{l=0}^{m} a_{k l}(\theta) s^{k} e^{s \tau_{l}},
$$

where $0<\tau_{0}<\tau_{1}<\cdots<\tau_{m}$ and $a_{k l}(\theta)$ are the coefficients of a polynomial with a finite set of bounded uncertainties, which depend on the uncertainties $\theta$. The quasi-polynomial (37) is exactly rewritten as a polytope whose interpolating functions exhibit mutual dependency. Therefore, the stability analysis of this type of polytopes implies the stability analysis of a class of nonlinear TDS, see [173, 174].

On the contrary and to finish this section, it is worth mentioning that some members of the scientific community have preferred to employ transformations, approximation methods or pseudo-delays to avoid the transcendental terms in the stability analysis of a TDS instead of using the direct methods presented above. Although, in many occasions, a direct approach to analyze the stability of a TDS is more efficient [202]. Among these, they can be found the Smith predictors [203], Rekasius transformation [204], and Padé approximation [205]. The Smith predictors allow to use a controller structure which takes the delay out of the control loop, which reduce the stability analysis to the one of a free-delay system. The employment of the Rekasius transformation implies an infinity-to-one holographic mapping (the mapping is asymmetric), and it is also impossible to track all of the infinitely many roots, especially, since the dominant root cannot be declared, as mentioned in [206]. The Padé approximation has been used to approximate the exponential function $e^{-s \tau}, s \in C$, through rational approximation of the form $\left(P_{m n}(s \tau) / P_{n m}(s \tau)\right)$, where

$$
\begin{aligned}
& P_{m n}(s \tau)=\sum_{j=0}^{m} \frac{(m+n-j) ! m !}{j !(m-j) !}(-s \tau)^{j}, \\
& P_{n m}(s \tau)=\sum_{j=0}^{n} \frac{(m+n-j) ! n !}{j !(n-j) !}(s \tau)^{j},
\end{aligned}
$$

see [207].

3.3. Stability in the Time Domain. In this section, a brief description of the most well-known criteria for the stability analysis of TDS in the time domain will be given. Emphasize the results using two types of Lyapunov-Krasovskii functionals: reduced type and complete type. While the first type of functional is usually the favorite of the scientific community, perhaps due to the relative flexibility to propose the functional candidate and to accomplish the requirements of the system studied, it seems that this type of functional only can provide sufficient conditions of stability and stabilization. The second type of functional is used by a narrowed community, perhaps due to the relative complexity compared to the first, but this has been shown to be closer to obtaining the necessary and sufficient conditions of stability and stabilization, see [208].

This approach is based primarily on two methods: the use of Lyapunov-Krasovskii (L-K) functional [146] or Lyapunov-Razumikhin (L-R) functions [145]. Both methods are an extension of the Lyapunov direct method [42] for free-delay systems. These stability criteria usually provide sufficient stability conditions in terms of linear matrix inequalities (LMIs), [209], which can be effectively solved by means of convex optimization techniques [210]. Although these two methods have received a great deal of attention, the results only offer conservative and sufficient stability conditions. The Razumikhin results allow one to obtain stability results based on adapted Lyapunov functions to analyze the stability of the TDS, while the Krasovskii results employ Lyapunov functionals as a natural extension to TDS. Despite the two methods provide interesting results for stability studies, the last method is the predominant one in research.

The main idea of Krasovskii consists in proposing an appropriate functional which can satisfy extensions of concepts and criteria of Lyapunov for TDS. One of these stability concepts is the definition, while the most used criterion is given below.

Theorem 3 (see [122]). Consider the TDS given in (11) and that there are $u, v, w: R^{+} \longrightarrow R^{+}$continuous nondecreasing functions, where $u(\alpha)$ and $v(\alpha)$ are positive for $\alpha>0$ and $u(0)=v(0)=0$.

(i) If there exists a continuous differentiable functional $V: R^{+} \times \mathbb{C} \longrightarrow R^{+}$such that

$$
\begin{aligned}
u(|\phi(0)|) & \leq V(t, \phi) \leq v\left(|\phi|_{\tau}\right), \\
\dot{V}(t, \phi) & \leq-w(|\phi(0)|),
\end{aligned}
$$

then the trivial solution of (11) is uniformly stable.

(ii) If the trivial solution of (11) is uniformly stable, and $w(\alpha)>0$ for $\alpha>0$, then the trivial solution of (11) is uniformly asymptotically stable.

(iii) If the trivial solution of (11) is uniformly asymptotically stable and if $\lim _{\alpha \longrightarrow \infty} u(\alpha)=$, then the trivial solution of (11) is globally uniformly asymptotically stable.

Consider the LTI-TDS of the form:

$$
\dot{x}(t)=A_{0} x(t)+A_{1} x(t-\tau),
$$

where $A_{0}$ and $A_{1}$ are matrices of appropriate dimensions. The functional ones proposed to satisfy the above conditions 
are known as L-K functional candidates and their basic form is as follows:

$$
V\left(x_{t}\right)=x^{T}(t) P x(t)+\int_{t-\tau}^{t} x^{T}(\alpha) Q x(\alpha) \mathrm{d} \alpha,
$$

where $P=P^{T}>0$ and $Q=Q^{T}>0$ are positive definite matrices (symmetric matrices where every eigenvalue is positive). This functional satisfies the conditions of Theorem 3 if there are $P>0$ and $Q>0$ which satisfy $A_{0}^{T} P+P A_{0}+P A_{1} Q^{-1} A_{1}^{T} P+Q<0$ or

$$
\left(\begin{array}{cc}
A_{0}^{T} P+P A_{0}+Q & P A_{1} \\
* & -Q
\end{array}\right)<0 .
$$

If this is true, then the functional is called L-K functional. However, this type of $\mathrm{L}-\mathrm{K}$ functional only provides delayindependent sufficient stability conditions for LTI-TDS with one delay, namely, sufficient conditions that can only be applied to LTI-TDS that are stable for all $\tau>0$. When the primary objective is to propose $\mathrm{L}-\mathrm{K}$ functional candidates that provide delay-dependent stability conditions and that these conditions may be necessary and sufficient conditions to determine stability in a TDS, as well as for linear freedelay systems. Unfortunately, the above is still an open problem of the TDS. Therefore, most of the research studies carried out focus not only on the type of L-K functional, but also on mathematical properties that reduce the conservatism of the stability conditions (LMI-based stability conditions) and/or these can relax the conditions. Some of these properties follow immediately.

Lemma 1 (Schur complement). Consider a given symmetric matrix $S=\left(\begin{array}{cc}S_{11} & S_{12} \\ * & S_{22}\end{array}\right)$, where $S_{11} \in R^{r \times r}$; then, the following conditions are equivalent:

$$
\begin{array}{r}
S<0, \\
S_{22}<0, \\
S_{11}-S_{12}^{T} S_{22}^{-1} S_{12}<0, \\
S_{11}<0, \\
S_{22}-S_{12}^{T} S_{11}^{-1} S_{12}<0 .
\end{array}
$$

Lemma 2. Let $Q=Q^{T}, H$ and $E$ given matrices with appropriate dimensions. Then, $Q+H F(t) E+E^{T} F^{T}(t) H^{T}<0$ holds for all $F(t)$ such that $F^{T}(t) F(t) \leq I$ if and only if there exists $\epsilon>0$ such that $Q+\epsilon^{-1} H H^{T}+\epsilon E^{T} E<0$.

Lemma 3 (Jensen inequality, see [166]). For any constant matrix $M \in R^{m \times m}, M=M^{T}>0$, scalar $\gamma>0$, vector function $\omega:[0, \gamma] \longrightarrow R^{m}$ such that the integrations concerned are well defined, then

$$
\gamma \int_{0}^{\gamma} \omega^{T}(\beta) M \omega(\beta) \mathrm{d} \beta \geq\left(\int_{0}^{\gamma} \omega(\beta) \mathrm{d} \beta\right)^{T} M\left(\int_{0}^{\gamma} \omega(\beta) \mathrm{d} \beta\right) .
$$

Lemma 4 (see [122]). Let $A, D, E, F$, and $P$ be real matrices with appropriate dimensions, and let $F^{T} F \leq I$ and $P>0$. Then, the following propositions are true:

(i) For any $x, y \in R^{n}, 2 x^{T} y \leq x^{T} P^{-1} x+y^{T} P y$

(ii) For any $x, y \in R^{n}$ and any $\epsilon>0$, $2 x^{T} D F E y \leq \varepsilon-1 x^{T} D D^{T} x+\varepsilon y^{T} E^{T} E y$

(iii) For any $\epsilon>0$ satisfying $P-\epsilon D D^{T}>0$, $(A+D F E)^{T} P^{-1}(A+D F E) \leq \varepsilon^{-1} E^{T} E+$ $A^{T}\left(P-\varepsilon D D^{T}\right)^{-1} A$

For decades the TDS research community has proposed different types of $\mathrm{L}-\mathrm{K}$ functional candidates and/or also different mathematical properties in order to satisfy the postulated in (iii) of Theorem 3.3. One of the most observed trends is to add quadratic or cross terms to the functional candidate as follows.

Consider the following L-K functional candidate:

$$
V\left(x_{t}\right)=x^{T}(t) \operatorname{Px}(t)+\sum_{j=1}^{r} V_{j}\left(x_{t}\right)
$$

where each term $V_{j}\left(x_{t}\right), j=1,2, \ldots, r$ can be of the form: cross terms $x^{T}(t) \int_{-\tau}^{0} \mathrm{Q} x(t+\alpha) \mathrm{d} \alpha$, quadratic terms $\int_{-\tau}^{0} \int_{t+\alpha}^{t}$ $x^{T}(\alpha) R x(\alpha) \mathrm{d} \alpha \mathrm{d} t$, quadratic terms for exponential terms $\int_{-\tau}^{0} x^{T}(t+\alpha) e^{2 \sigma \alpha} Q x(t+\alpha) \mathrm{d} \alpha$, cross terms $x^{T}(t) \int_{-\tau}^{0} Q(\alpha) x$ $(t+\alpha) \mathrm{d} \alpha$, and quadratic terms $\int_{-\tau}^{0} \int_{-\tau}^{0} x^{T}(t+\alpha) R(\alpha, \beta) x$ $(\alpha+\beta) \mathrm{d} \alpha \mathrm{d} \beta$.

Typically, these terms (quadratic or crossed) are introduce depending on the type of system analyzed (RFDE, DFDE, and NFDE, among others) or the type of conditions required to obtain (robustness and exponential estimates, among others) as an effort to obtain the functional one that grants less restrictive conditions and more types of systems can be analyzed. Also, comparisons between various criteria of delay-dependent stability can be observed in the literature, to demonstrate the efficiency and loss of conservatism with the proposed conditions, see [211]. In this context, there are miles of contributions giving necessary conditions of stability, among which the following can be mentioned [39, 212-238]. In most of these contributions a type of functional known as reduced type functional is used. However, until now it is unknown what type of reduced-type functional is suitable for the type of TDS analyzed, [214, 239-241]. Therefore, some criteria for the construction of full size (complete) type L-K functionals have been developed with the intention of solving these problems.

The construction of the complete-type functional requires a prior proposal of the quadratic derivative of the functional and the construction of the so-called delay Lyapunov matrix. The first results were proposed in [242], followed by some interesting results such as those given in $[243,244]$ for RFDE with one delay, while in [245] some of the results have been extended to a general case of LTI-TDS. In the latter, it is also shown that the constructed functional requires additional information to admit a lower quadratic bound. In [213], an interesting numerical scheme for the construction of complete type L-K functionals has been 
proposed using the LMI approach. In [246, 247], properties are clarified and completed for the construction of a functional with upper and lower quadratic bounds. This technique can be summarized in the following result.

Theorem 4 (see [123]). Consider a prescribed quadratic functional of the form:

$$
\omega\left(x_{t}\right)=\omega_{1}\left(x(t), W_{1}\right)+\omega_{2}\left(x_{t}, W_{2}\right)+\omega_{3}\left(x(t-\tau), W_{3}\right),
$$

where $\omega_{1}\left(x(t), W_{1}\right)=x^{T}(t) W_{1} x(t)$,

$$
\begin{aligned}
\omega_{1}\left(x_{t}, W_{2}\right) & =\int_{\tau}^{0} x^{T}(t+\alpha) W_{2} x(t+\alpha) \mathrm{d} \alpha, \\
\omega_{2}\left(x(t-\tau), W_{3}\right) & =x^{T}(t-\tau) W_{3} x(t-\tau),
\end{aligned}
$$

and $W_{1}, W_{2}$, and $W_{3}$ are positive definite matrices of appropriate dimensions. If the LTI-TDS (40) is stable, then there is only one functional $v\left(x_{t}\right)$ such that

$$
\dot{v}\left(x_{t}\right)=-\omega\left(x_{t}\right) \text {. }
$$

This functional is known as complete type L-K functional and it is given by

$$
\begin{aligned}
v\left(x_{t}\right)= & x^{T}(t) U(0) x(t)+2 x^{T}(t) \int_{-\tau}^{0} U(-\tau-\alpha) A_{1} x(t+\alpha) d \alpha+\int_{-\tau}^{0} \int_{-\tau}^{0}\left[x^{T}\left(t+\alpha_{1}\right) A_{1}^{T} U\left(\alpha_{1}-\alpha_{2}\right) A_{1} x\left(t+\alpha_{2}\right)\right] d \alpha_{1} d \alpha_{2} \\
& +\int_{-\tau}^{0} x^{T}(t+\alpha)\left[W_{1}+(\tau+\alpha) W_{2}\right] x(t+\alpha) d \alpha
\end{aligned}
$$

where

$$
U(\varsigma)=\int_{0}^{\infty} K^{T}(t) W K(t+\varsigma) \mathrm{d} t, \quad \varsigma \in R,
$$

is a counterpart of the classical Lyapunov matrix equation in the context of Lyapunov quadratic forms for the linear delayfree systems; therefore, it is called Lyapunov matrix for TDS (delay Lyapunov matrix). Here, $0<W=W_{0}+W_{1}+$ $\tau W_{2} \in R^{n \times n}$ and $K(t): R \longrightarrow R^{n \times n}$ is the fundamental matrix of LTI-TDS (40) and the solution of the matrix equation:

$$
\dot{K}(t)=A_{0} K(t)+A_{1} K(t-\tau), \quad t \geq 0,
$$

with initial condition $K(t)=0$ for $t<0$ y $K(0)=I_{n}$. In addition, the delay Lyapunov matrix satisfies the following conditions:

$$
U^{\prime}(\varsigma)=U(\varsigma) A_{0}+U(\varsigma-\tau) A_{1}, \quad \varsigma \geq 0,
$$

$$
\begin{aligned}
& U(\varsigma)=U^{T}(-\varsigma), \quad \varsigma \geq 0, \\
& -W=U(0) A_{0}+A_{0}^{T} U(0)+U^{T}(\tau) A_{1}+A_{1}^{T} U(\tau) .
\end{aligned}
$$

Equations (52)-(54) are known as dynamic property, symmetry property, and algebraic property, respectively.

One of the key points to use this type of functional are the existence, uniqueness, and numerical calculation of the delay Lyapunov matrix (50), for which several manuscripts have been dedicated to this sense, see [248-254]. Some of the functionals prescribed $w\left(x_{t}\right)$ to construct complete type L-K functionals $v\left(x_{t}\right)$ are as follows.

(i) For LTI-RFDE [255],

$$
\omega\left(x_{t}\right)=x^{T}(t) W_{1} x(t)+2 x^{T}(t) Z A_{-} 1 x(t-\tau)+x^{T}(t-\tau) W_{2} x(t-\tau)+\int_{\tau}^{0} x^{T}(t+\alpha) W_{3} x(t+\alpha) \mathrm{d} \alpha .
$$

(ii) For LTI-DFDE [256],

$$
\begin{aligned}
\omega\left(x_{t}\right)= & x^{T}(t) W_{1} x(t)+x^{T}(t-\tau) W_{2} x(t-\tau)+\int_{\tau}^{0} x^{T}(t+\alpha) W_{3} x(t+\alpha) d \alpha+2 x^{T}(t) W_{4} x(t-\tau) \\
& +2 x^{T}(t) \int_{\tau}^{0} W_{5} x(t+\alpha) \mathrm{d} \alpha+2 x^{T}(t-\tau) \int_{\tau}^{0} W_{6} x(t+\alpha) \mathrm{d} \alpha .
\end{aligned}
$$


(iii) For RFDE with time-varying delay [257],

$$
\omega\left(x_{t}\right)=x^{T}(t) W_{1} x(t)+\dot{x}^{T}(t) W_{2} \dot{x}(t) .
$$

(iv) For RFDE with uncertain coefficients $H \Delta A_{0}$ and $H \Delta A_{1}$ and an uncertain time-varying delay $\tau(t)=h_{1}+\eta_{1}(t)$, with $\quad h_{1} \in R^{+} \quad$ and $|\eta(t)| \leq \mu_{1} \in R^{+}[258]$,

$$
\begin{aligned}
\omega\left(x_{t}\right)= & x^{T}(t) W_{1} x(t)-2\left[x^{T}(t) U^{T}(0)+\int_{-h_{1}}^{0} x^{T}(t+\alpha) A_{1}^{T} U\left(h_{1}+\alpha\right) \mathrm{d} \alpha\right] \times\left[H \Delta A_{0} x(t)+H \Delta A_{1} x\left(t-h_{1}\right)-H \Delta A_{1}\right. \\
& \left.\cdot \int_{t-h_{1}-\eta_{1}}^{t-h_{1}} \dot{x}(\alpha) \mathrm{d} \alpha-A_{1} \int_{t-h_{1}-\eta_{1}}^{t-h_{1}} \dot{x}(\alpha) \mathrm{d} \alpha\right] .
\end{aligned}
$$

This type of functional and the delay Lyapunov matrix are giving good results for the design of control laws such as linear quadratic suboptimal controllers [259]; recently, these have also been used to designing dynamic stabilizing controllers (predictor-based controls) for preserving the exponential stability of the closed-loop system after the replacement of the integrals by finite sums [260-263], robotic systems with constant input time delay through the active disturbance rejection paradigm and generalized proportional integral observers [264], design of delayed output-feedback controllers that optimize a quadratic cost function [265], necessary and sufficient exponential stability condition for systems with multiple delays [266], and partial differential equations [267]. Furthermore, these types of functionals can provide necessary and sufficient stability conditions for linear systems with pointwise and distributed delays [208]. Here, a stability criterion is presented for the exponential stability of systems with multiple point and distributed delays. These conditions are in terms of the delay Lyapunov matrix (50) using the evaluation of a complete type L-K functionals at a pertinent initial function that depends on the system fundamental matrix. Undoubtedly, this topic is solving important open problems in the area of TDS, whereby it is highly recommended to direct current and future research.

3.4. Numerical Methods (Applications). One of the most important aspects in the stability analysis of time-delay linear systems is the calculation of characteristic roots of the linear/linearized dynamics, being the root with the maximum real part a very important one [268]. The characteristic equation is a quasi-polynomial equation whose roots are computed through a numerical method for nonlinear equations.

In the contribution of Engelborghs [269], it is proposed a Matlab package for numerical bifurcation analysis of TDS, and the computation of the rightmost characteristic roots is carried out by using a linear multistep method (LMS method) [270].

The monograph of Breda et al. [271] presents a comprehensive set of pseudospectral techniques (Pseudospectral Differentiation Method and Piecewise Pseudospectral Differentiation Method) to analyze the stability of the solution of linear TDS with numerical implementations in Matlab.
In [272], Louisell establishes a method for determining the stability exponent and eigenvalue abscissas of a linear delay system based on examining the endpoint values of the solution to a functional equation occurring in the Lyapunov theory of delay equations. Other interesting related works are $[273,274]$. In $[275,276]$, it is presented a methodology for calculating the Lyapunov matrix with a distributed delay, whose algorithm consist in solving a two points boundary value problem for a delay-free system.

Olgac and Sipahi provide an alternative procedure based on the cluster treatment of characteristic roots to analyze the stability of multiple time-delayed LTI dynamics. This methodology detects all the stable regions precisely, in the space of the time delays, by means of a set of curves (kernel and offspring) which count the possible imaginary root crossings for the system (see [206, 277-282] and references therein for a comprehensive treatment).

Concerning the eigenvalue problem, Michiels has come up with different procedures. In [283], it is provided a characterization of the solutions to an arbitrary nonlinear eigenvalue problem as the reciprocal eigenvalues of an infinite-dimensional operator, and the resulting algorithm is completely equivalent to the standard Arnoldi method, including many of its properties. An extension of last contribution [284] is used to compute the partial Schur factorization of a nonlinear eigenvalue problem. In [285], it is presented a procedure to compute solutions to a type of nonlinear eigenvalue problem with low-rank structure. The algorithm turns out to be equivalent to the Arnoldi method (even in the numerical behavior). In [286], first, the formula for the sensitivity of a simple eigenvalue with respect to a variation of a parameter is extended to the case of multiple non-semisimple eigenvalues. Also, it is provided a dual treatment of the delay eigenvalue problem, in one hand, at the level of the finite-dimensional nonlinear eigenvalue problem and, on the other hand, at the level of a standard operator eigenvalue problem. A numerical procedure to compute the pseudospectral abscissa is given in [287], whose main feature is that the approach is a pioneering applicable procedure for nonlinear problems.

The most complex systems have nonlinearities and delays, which can be easy to notice or sometimes not so much. A delay in a system is a phenomenon that can be seen as the dead time between transmitting and executing an action. In 
this context, delays are due to the fact that system dynamics are associated with past events. In general, delays are undesirable phenomena in a system because they can instabilize or produce a poor performance in their response. However, in recent years, it has been shown that delays can also favor stabilizing and improving the performance of the system [288, 289].

The deliberate application of delays to stabilize systems is a latent topic in the literature, one of the most important contributions in this aspect is found in [290] that drove a whole stream of research in what is known as time-delayed feedback control (TDFC). The technique is to deliberately introduce delays into the control scheme to $\sigma$-stabilize a system with or without inherent delays [291-308]. This is due to the advantages that they have in applications on experimental platforms, such as noise attenuation, nonimplementation of estimators, observers and speed sensors, avoiding filters, as well as its easy implementation. In addition, the $\sigma$-stability analysis or $\sigma$-stability regions allows a fragility analysis of the controllers gains, which can give a measure of the robustness of the closed-loop system under variations of the controller gains, see [303, 309-311].

\section{Advances in Stability and Stabilization of Nonlinear Time-Delay Systems}

In recent years, different concepts have emerged that are less restrictive to classical concepts to determine stability in nonlinear time-delay systems. Next, some of them are mentioned.

4.1. Complex Delay Complex Networks. One topic which has attracted attention from the scientific community is the analysis and control of complex networks [312, 313], which describe a wide variety of physical and social systems [314], from population interactions, brain activity, and language patterns to Internet traffic behavior among other interesting phenomena. One control problem derived from the complex systems control is the synchronization of delay coupled networks $[315,316]$. In this sense, a formal stability analysis for the synchronization of complex networks, in particular for a set of oscillators, is usually given for linearized systems in a vicinity of the equilibrium point [317] by computing the stability regions in the delay parameters or using the circle criterion [318]. Some reported contributions can be found in $[169,238,319-328]$.

4.2. Robotic Teleoperation and Predictor-Based Control. Robotic teleoperation and haptic teleoperation has been a highly active topic in last years, specially now with the active development of virtual reality and haptic interfaces [329-334]. The time delay in these applications arises mainly to the latency aspects of the virtual reality system [335-338], and the network traffic in a teleoperation system [339-342].

The stability analysis for teleoperation has the following general approaches: (i) The use of predictors working on the stability of the system in the feedforward dynamics with nominal or robust criteria, see [203,343-360]

(ii) Passivity-based approaches whose stability tests can be given in the Lyapunov-Krasovskii sense (see [106, 342, 361-374])

(iii) Robust and predictor-based schemes whose stability is given for time-delay dynamics tested by means of time- or frequency-based approaches [264, 375-378]

(iv) Other schemes which assume the delay as a disturbance to be compensated by robust or adaptive techniques [379-384]

4.3. Nonlinear and Fractional Order Systems. Lyapunov methods have shown very useful for the study of stability and the design of nonlinear control laws [130, 385, 386]. Here, discontinuous [387] and fractional order systems [388] represent an important challenge. Particularly, sliding mode control techniques is an active field of research where Lyapunov methods have been a key factor [389-393]. The main advantages of sliding mode control, including robustness and finite time convergence, are supported by no conventional Lyapunov methods.

On the contrary, fundamental research has shown that a large variety of physical signals can be described by means of functions with more varied topological properties [394-397], which can be continuous but not necessarily differentiable in any integer-order sense [398]. This has motivated the design of fractional order sliding mode-based controllers which have been proved to be robust against Hölder disturbances [399].

Fractional calculus has become an emerging approach for modeling complex systems, which has attracted the attention of several areas of study, including control systems. The use of fractional versions of PID controllers [400-406] has increased the interest and development of fractional control designs. As a natural consequence, this approach has been extended to a wider class of systems, especially time delay ones which has come up with stability studies and analyses.

Since classic methods for stability testing integer systems such as Routh-Hurwitz are not universally valid, the stability analysis for even linear fractional systems is more challenging. In particular, among the approaches of stability study for fractional time-delay systems, some pioneering contributions are given by Hwang and Cheng. In one hand, the Lambert $\mathrm{W}$ function is proposed for the stability analysis [407] and, on the other hand, in [408], the characteristic equation of the system is numerically analyzed for a BIBO stability test by means of Cauchy's integral theorem. In [409], a numerical procedure to obtain the delay values where there is a root crossing (from the left half plane to the right half and vice versa), for a further procedure of finding the stability zones in terms of the delay value. Other fundamental contributions are given in [410-415]. In [416], a Matlab toolbox for the $H_{\infty}$-stability analysis of fractional 
systems with commensurate delays is provided. L-K stability approaches for fractional systems are given in [417-419]. In $[325,420-424]$, some L-K stability approaches are given in the realm of neural network framework.

Concerning using Smith predictor-based stabilizing controllers, several applications are reported, see [425-432].

\section{Conclusions}

This paper has presented a set of criteria concerning robust stability of dynamical systems with or without delay, which is important for the analysis of complex systems which may not provide complete information, involving parameter uncertainties. On the one hand, the ideas of robust stability through families of polynomials were addressed and the main criteria discussed. On the other hand, an emerging topic in the area of complex systems such as time-delay systems stability was analyzed, including the motivation, basic results for its understanding, the difficulties involved with respect to systems without delay, the time and frequency approaches, applications involving complex oscillators, current trends in this field of research, and some interesting open problems. This review explored the stability as a fundamental structural property which is crucial in the analysis and development of studies and applications of complex dynamical systems, in which the couplings and dynamical behavior may come up with new developments in a wide variety of research areas.

\section{Data Availability}

No data were used to support this study.

\section{Conflicts of Interest}

The authors declare that there are no conflicts of interest regarding the publication of this paper.

\section{Acknowledgments}

In memory of Oscar Villafuerte-Segura, my brother, my partner, and unconditional friend. I will never forget you. This work was partially supported by Secretaría de Investigación y Posgrado-IPN under grant SIP20201675 (A. Luviano-Juárez).

\section{References}

[1] G. A. Pagani and M. Aiello, "Power grid complex network evolutions for the smart grid," Physica A: Statistical Mechanics and its Applications, vol. 396, pp. 248-266, 2014.

[2] C.-C. Chu and H. H.-C. Iu, "Complex networks theory for modern smart grid applications: a survey," IEEE Journal on Emerging and Selected Topics in Circuits and Systems, vol. 7, no. 2, pp. 177-191, 2017.

[3] W. Yu, G. Wen, X. Yu, Z. Wu, and J. Lü, "Bridging the gap between complex networks and smart grids," Journal of Control and Decision, vol. 1, no. 1, pp. 102-114, 2014.

[4] F. Dörfler, M. Chertkov, and F. Bullo, "Synchronization in complex oscillator networks and smart grids," Proceedings of the National Academy of Sciences, vol. 110, no. 6, pp. 2005-2010, 2013.

[5] S. Wollenberg and F Bruce, "Toward a smart grid: power delivery for the 21st century," IEEE Power and Energy Magazine, vol. 3, no. 5, pp. 34-41, 2005.

[6] M. Xue, W. Wang, and S. Roy, "Security concepts for the dynamics of autonomous vehicle networks," Automatica, vol. 50, no. 3, pp. 852-857, 2014.

[7] D. J. Stilwell, E. M. Bollt, and D. G. Roberson, "Sufficient conditions for fast switching synchronization in timevarying network topologies," SIAM Journal on Applied Dynamical Systems, vol. 5, no. 1, pp. 140-156, 2006.

[8] L. Iftekhar and R. Olfati-Saber, "Autonomous driving for vehicular networks with nonlinear dynamics," in Proceedings of the 2012 IEEE Intelligent Vehicles Symposium, pp. 723729, Madrid, Spain, June 2012.

[9] C. J. E. Naranjo, R. Garcia, and T. de Pedro, "Lane-change fuzzy control in autonomous vehicles for the overtaking maneuver," IEEE Transactions on Intelligent Transportation Systems, vol. 9, no. 3, pp. 438-450, 2008.

[10] E. Frazzoli, M. A. Dahleh, and E. Feron, "Real-time motion planning for agile autonomous vehicles," Journal of Guidance, Control, and Dynamics, vol. 25, no. 1, pp. 116-129, 2002.

[11] B. H. Junker and F. Schreiber, Analysis of Biological Networks, John Wiley \& Sons, Hoboken, New Jersey, USA, 2011.

[12] R.-S. Wang, S. Zhang, Y. Wang, X.-S. Zhang, and L. Chen, "Clustering complex networks and biological networks by nonnegative matrix factorization with various similarity measures," Neurocomputing, vol. 72, no. 1-3, pp. 134-141, 2008.

[13] E. Estrada, "Generalized walks-based centrality measures for complex biological networks," Journal of Theoretical Biology, vol. 263, no. 4, pp. 556-565, 2010.

[14] P. Wang, L. ü. Jinhu, and X. Yu, "Identification of important nodes in directed biological networks: a network motif approach,” PLoS One, vol. 9, no. 8, 2014.

[15] M. Rosvall and C. T. Bergstrom, "Maps of random walks on complex networks reveal community structure," Proceedings of the National Academy of Sciences, vol. 105, no. 4, pp. 1118-1123, 2008.

[16] R. Pastor-Satorras, C. Castellano, P. Van Mieghem, and A. Vespignani, "Epidemic processes in complex networks," Reviews of Modern Physics, vol. 87, no. 3, pp. 925-979, 2015.

[17] D. Aguilar-Velázquez and L. Guzmán-Vargas, "Critical synchronization and $1 / \mathrm{f}$ noise in inhibitory/excitatory richclub neural networks," Scientific Reports, vol. 9, no. 1, pp. 1-13, 2019.

[18] A. Yazdani and P. Jeffrey, "Robustness and vulnerability analysis of water distribution networks using graph theoretic and complex network principles," in Proceedings of the 12th Annual Conference on Water Distribution Systems Analysis (WDSA), pp. 933-945, Tucson, AR, USA, September 2010.

[19] C. Ocampo-Martinez, V. Puig, G. Cembrano, and J. Quevedo, "Application of predictive control strategies to the management of complex networks in the urban water cycle applications of control," IEEE Control Systems Magazine, vol. 33, no. 1, pp. 15-41, 2013.

[20] R. Juárez, I. Y. Fernández, and L. Guzmán, "Characteristics of the oil transport network in the south of Mexico," Journal of Physics: Conference Series, vol. 792, Article ID 012096, 2017. 
[21] Z. Ma, S. M. Jeter, and S. I. Abdel-Khalik, "Flow network analysis application in fuel cells," Journal of Power Sources, vol. 108, no. 1-2, pp. 106-112, 2002.

[22] Q. Ji, H.-Y. Zhang, and Y. Fan, "Identification of global oil trade patterns: an empirical research based on complex network theory," Energy Conversion and Management, vol. 85, pp. 856-865, 2014.

[23] M. Wang, Y. Chen, L. Tian, S. Jiang, Z. Tian, and R. Du, "Fluctuation behavior analysis of international crude oil and gasoline price based on complex network perspective," Applied Energy, vol. 175, pp. 109-127, 2016.

[24] K. Klemm, V. M. Eguíluz, R. Toral, and M. San Miguel, "Nonequilibrium transitions in complex networks: a model of social interaction," Physical Review E, vol. 67, no. 2, Article ID 026120, 2003.

[25] L. Guzmán-Vargas and R. Hernández-Pérez, "Small-world topology and memory effects on decision time in opinion dynamics," Physica A: Statistical Mechanics and its Applications, vol. 372, no. 2, pp. 326-332, 2006.

[26] M. Kitsak, L. K. Gallos, F. S. Havlin et al., "Identification of influential spreaders in complex networks," Nature Physics, vol. 6, no. 11, pp. 888-893, 2010.

[27] A.-L. Albert and R. Albert, "Emergence of scaling in random networks," Science, vol. 286, no. 5439, pp. 509-512, 1999.

[28] H. Zhang, N. Liu, X. Chu et al., "Network slicing based 5G and future mobile networks: mobility, resource management, and challenges," IEEE Communications Magazine, vol. 55, no. 8, pp. 138-145, 2017.

[29] A. E. Motter and Y.-C. Lai, "Cascade-based attacks on complex networks," Physical Review E, vol. 66, no. 6, Article ID 065102, 2002.

[30] R. Srikant, The Mathematics of Internet Congestion Control, Springer Science \& Business Media, Berlin, Germany, 2004.

[31] R. Handcock, D. Swain, G. Bishop-Hurley et al., "Monitoring animal behaviour and environmental interactions using wireless sensor networks, GPS collars and satellite remote sensing," Sensors, vol. 9, no. 5, pp. 3586-3603, 2009.

[32] D. M. P. Jacoby, Y. P. Papastamatiou, and R. Freeman, "Inferring animal social networks and leadership: applications for passive monitoring arrays," Journal of The Royal Society Interface, vol. 13, no. 124, Article ID 20160676, 2016.

[33] A. G. Villa, A. Salazar, and F. Vargas, "Towards automatic wild animal monitoring: identification of animal species in camera-trap images using very deep convolutional neural networks," Ecological informatics, vol. 41, pp. 24-32, 2017.

[34] R. Vera-Amaro, M. E. Rivero-Ángeles, and A. Luviano-Juárez, "Data collection schemes for animal monitoring using WSNSassisted by UAVS: Wsns-oriented or UAV-oriented," Sensors, vol. 20, no. 1, p. 262, 2020.

[35] Y.-Y. Liu, J.-J. Slotine, and A.-L. Barabási, "Controllability of complex networks,” Nature, vol. 473, no. 7346, pp. 167-173, 2011.

[36] B. D. MacArthur, R. J. Sánchez-García, and J. W. Anderson, "Symmetry in complex networks," Discrete Applied Mathematics, vol. 156, no. 18, pp. 3525-3531, 2008.

[37] A. J. Whalen, S. N. Brennan, T. D. Sauer, and S. J. Schiff, "Observability and controllability of nonlinear networks: the role of symmetry," Physical Review X, vol. 5, no. 1, Article ID 011005, 2015.

[38] D. Leitold, Á. Vathy-Fogarassy, and J. Abonyi, "Controllability and observability in complex networks-the effect of connection types," Scientific Reports, vol. 7, no. 1, pp. 1-9, 2017.
[39] S.-I. Niculescu, "On delay-dependent stability under model transformations of some neutral linear systems," International Journal of Control, vol. 74, no. 6, pp. 609-617, 2001.

[40] V. L. Kharitonov, "Asymptotic stability of an equilibrium position of a family of systems of linear differential equations," Differentsial'nye Uravneniya, vol. 14, pp. 2086-2088, 1978.

[41] D. McFarlane and K. Glover, "A loop-shaping design procedure using H/sub infinity/synthesis," IEEE Transactions on Automatic Control, vol. 37, no. 6, pp. 759-769, 1992.

[42] A. Liapounoff, "Problème général de la stabilité du mouvement (in French)," Annales de la Faculté des sciences de Toulouse: Mathématiques, vol. 9, pp. 203-474, 1907.

[43] J. C. Maxwell, "I. On governors," Proceedings of the Royal Society of London, vol. 16, pp. 270-283, 1868.

[44] J. L. de La Grange, Méchanique Analytique, Veuve Desaint, 1788.

[45] J. L. Lagrange, "Sur l'altération des moyens mouvements des planetes mem," Acad. Sci. Berlin, vol. 199, 1776.

[46] G. L. Dirichlet, "Über die stabilität des gleichgewichts," Journal für die reine und angewandte Mathematik, vol. 32, pp. 85-88, 1846.

[47] B. Brogliato, "Absolute stability and the Lagrange-Dirichlet theorem with monotone multivalued mappings," Systems \& Control Letters, vol. 51, no. 5, pp. 343-353, 2004.

[48] R. I. Leine, "The historical development of classical stability concepts: Lagrange, Poisson and Lyapunov stability," Nonlinear Dynamics, vol. 59, no. 1-2, p. 173, 2010.

[49] S. P. Bhattacharyya and L. H. Keel, "Robust control: the parametric approach," in Advances in Control Education, Elsevier, Amsterdam, Netherlands, 1995.

[50] D. Hinrichsen and A. J. Pritchard, Mathematical Systems Theory I: Modelling, State Space Analysis, Stability and Robustness, Springer, Berlin, Germany, 2005.

[51] C. Hermité, "Le nombre des racines d'une équation algebraique comprises entre des limites données," Crelle's Journal, vol. 52, pp. 1-6, 1895.

[52] R. E. Bellman and K. L Cooke, Differential-difference Equations, Rand Corporation, Santa Monica, CA, USA, 1963.

[53] P. A. Fuhrmann and B. N. Datta, "On bezoutians, van der monde matrices, and the lienard-chipart stability criterion," Linear Algebra and its Applications, vol. 120, pp. 23-37, 1989.

[54] M. Fu and Y. Z. Tsypkin, "Modified Mikhailov plots for robust absolute stability with non-parametric perturbations and uncertain nonlinearity," International Journal of Control, vol. 58, no. 4, pp. 925-932, 1993.

[55] B. Ross Barmish and E. I. Jury, "New tools for robustness of linear systems," IEEE Transactions on Automatic Control, vol. 39, no. 12, p. 2525, 1994.

[56] R. Tempo, "A dual result to Kharitonov's theorem," IEEE Transactions on Automatic Control, vol. 35, no. 2, pp. 195198, 1990.

[57] K.-K. Young, P. Kokotovic, and V. Utkin, "A singular perturbation analysis of high-gain feedback systems," IEEE Transactions on Automatic Control, vol. 22, no. 6, pp. 931938, 1977.

[58] A. Ilchmann and E. P. Ryan, "High-gain control without identification: a survey," GAMM-Mitteilungen, vol. 31, no. 1, pp. 115-125, 2008.

[59] L. Levaggi, "High-gain feedback and sliding modes in infinite dimensional systems," Control and Cybernetics, vol. 33, no. 1, pp. 33-50, 2004. 
[60] H. Nogami, H. Maeda, S. Kodama, and M. Vidyasagar, "Robust stabilization of multivariable high gain feedback systems," Transactions of the Society of Instrument and Control Engineers, vol. 23, no. 4, pp. 364-370, 1987.

[61] J. Schumacher, "Almost stabilizability subspaces and high gain feedback," IEEE Transactions on Automatic Control, vol. 29, no. 7, pp. 620-628, 1984.

[62] Y. Z. Tsypkin and B. T. Polyak, "High-gain robust control," European Journal of Control, vol. 5, no. 1, pp. 3-9, 1999.

[63] J. L. Willems, "Disturbance isolation in linear feedback systems," International Journal of Systems Science, vol. 6, no. 3, pp. 233-238, 1975.

[64] J. Willems, "Almost invariant subspaces: an approach to high gain feedback design-part II: almost conditionally invariant subspaces," IEEE Transactions on Automatic Control, vol. 27, no. 5, pp. 1071-1085, 1982.

[65] B. Aguirre, C. Ibarra, and R. Suárez, "Sufficient algebraic conditions for stability of cones of polynomials," Systems \& Control Letters, vol. 46, no. 4, pp. 255-263, 2002.

[66] B. Aguirre and R. Suárez, "Algebraic test for the Hurwitz stability of a given segment of polynomials," Boletin de la Sociedad Matemática Mexicana, vol. 12, no. 3, pp. 261-275, 2006.

[67] B. Aguirre Hernández, J. Solis-Daun, and R. Suárez, "Stabilization of linear systems: a polynomial approach," in Advances in Dynamics, Instrumentation and Control, World Scientific, Singapore, 2007.

[68] S. Bialas, "A necessary and sufficient condition for the stability of convex combinations of stable polynomials or matrices," Bulletin of the Polish Academy of Sciences, vol. 33, pp. 473-480, 1985.

[69] B. S. Bollepalli and L. R. Pujara, "On the stability of a segment of polynomials," IEEE Transactions on Circuits and Systems I: Fundamental Theory and Applications, vol. 41, no. 12, pp. 898-901, 1994.

[70] N. K. Bose, "A system-theoretic approach to stability of sets of polynomials," Linear Algebra and its Role in Systems Theory, vol. 47, pp. 25-34, 1985.

[71] H. Bouguerra, B. C. Chang, H. H. Yeh, and S. S. Banda, "Fast stability checking for the convex combination of stable polynomials," IEEE Transactions on Automatic Control, vol. 35, no. 5, pp. 586-588, 1990.

[72] D. Hinrichsen and V. L. Kharitonov, "Stability of polynomials with conic uncertainty," Mathematics of Control, Signals, and Systems, vol. 8, no. 2, pp. 97-117, 1995.

[73] J.-A. Lopez-Renteria, B. Aguirre-Hernandez, and F. Verduzco, "The boundary crossing theorem and the maximal stability interval," Mathematical Problems in Engineering, vol. 2011, Article ID 123403, 2011.

[74] B. Ross Barmish and E. I. Jury, "New tools for robustness of linear systems," IEEE Transactions on Automatic Control, vol. 39, no. 12, p. 2525, 1994.

[75] M. Fu and B. Ross Barmish, "Maximal unidirectional perturbation bounds for stability of polynomials and matrices," Systems \& Control Letters, vol. 11, no. 3, pp. 173-179, 1988.

[76] S. P. Bhattacharyya and L. H. Keel, "Robust control: the parametric approach," in Advances in Control Education, Elsevier, Amsterdam, Netherlands, 1995.

[77] H. Chapellat and S. P. Bhattacharyya, "An alternative proof of Kharitonov's theorem," IEEE Transactions on Automatic Control, vol. 34, no. 4, pp. 448-450, 1989.

[78] N. K. Bose, "Tests for Hurwitz and Schur properties of convex combination of complex polynomials," IEEE
Transactions on Circuits and Systems, vol. 36, no. 9, pp. 1245-1247, 1989.

[79] C. Hwang and S.-F. Yang, "The use of Routh array for testing the Hurwitz property of a segment of polynomials," Automatica, vol. 37, no. 2, pp. 291-296, 2001.

[80] A. Rantzer, "Stability conditions for polytopes of polynomials," in Proceedings of the 29th IEEE Conference on Decision and Control, pp. 27-31, Honolulu, HI, USA, 1990.

[81] J. Ackermann, Robust Control: The Parameter Space Approach, Springer Science \& Business Media, Berlin, Germany, 2012.

[82] B. Aguirre-Hernández, F. R. García-Sosa, C. A. LoredoVillalobos, R. Villafuerte-Segura, and E. Campos-Cantón, "Open problems related to the Hurwitz stability of polynomials segments," Mathematical Problems in Engineering, vol. 2018, Article ID 2075903, 2018.

[83] N. K. Bose, "Argument conditions for Hurwitz and Schur polynomials from network theory," IEEE Transactions on Automatic Control, vol. 39, no. 2, pp. 345-346, 1994.

[84] T. Büyükköroğlu and V. Dzhafarov, "On ray properties of Hurwitz polynomials," IOSR Journal of Mathematics, vol. 12, no. 6, pp. 87-90, 2016.

[85] M. N. Góra, "Stability of the convex combination of polynomials," Control and Cybernetics, vol. 36, no. 2, pp. 425442, 2007.

[86] E. Zalot, "Criterions for the stability of the convex combination of polynomials," Automatyka/Akademia GórniczoHutnicza im. StanisŃawa Staszica w Krakowie, vol. 10, pp. 87-95, 2006.

[87] M.-T. Ming-Tzu Ho, A. Datta, and S. P. Bhattacharyya, "An elementary derivation of the Routh-Hurwitz criterion," IEEE Transactions on Automatic Control, vol. 43, no. 3, pp. 405409, 1998.

[88] S. Faedo, "A new stability problem for polynomials with real coefficients,”Ann. Scuola Norm. Sup. Fis. Sci. Mat. Ser., vol. 3, pp. 53-63, 1953.

[89] V. L. Kharnitonov, "Asymtotic stability of an equilibrium position of a family of systems of linear di ential equations," Differential Uravnen, vol. 14, pp. 2086-2088, 1978.

[90] N. Bose and Y. Shi, “A simple general proof of Kharitonov's generalized stability criterion," IEEE Transactions on Circuits and Systems, vol. 34, no. 10, pp. 1233-1237, 1987.

[91] M. Mansour and B. D. O. Anderson, "Kharitonov's theorem and the second method of Lyapunov," Systems \& Control Letters, vol. 20, no. 1, pp. 39-47, 1993.

[92] R. J. Minnichelli, J. J. Anagnost, and C. A. Desoer, “An elementary proof of Kharitonov's stability theorem with extensions," IEEE Transactions on Automatic Control, vol. 34, no. 9, pp. 995-998, 1989.

[93] K. Yeung and S. Wang, "A simple proof of Kharitonov's theorem," IEEE Transactions on Automatic Control, vol. 32, no. 9, pp. 822-823, 1987.

[94] V. L. Kharitonov, "Routh-Hurwitz problem for families of polynomials and quasi-polynomials," Matematicheskaia Fizika, vol. 1, no. 26, pp. 69-79, 1979.

[95] A. A. Kale and A. L. Tits, "On Kharitonov's theorem without invariant degree assumption," Automatica, vol. 36, no. 7, pp. 1075-1076, 2000.

[96] J. C. Willems and R. Tempo, "The Kharitonov theorem with degree drop," IEEE Transactions on Automatic Control, vol. 44, no. 11, pp. 2218-2220, 1999.

[97] T. Meressi, D. Chen, and B. Paden, "Application of Kharitonov's theorem to mechanical systems," IEEE Transactions on Automatic Control, vol. 38, no. 3, pp. 488-491, 1993. 
[98] F. Asadi, S. Pongswatd, K. Eguchi, and N. L. Trung, "Modeling uncertainties in DC-DC converters," Synthesis Lectures on Electrical Engineering, vol. 3, no. 2, pp. 1-292, 2018.

[99] K. Panneerselvam and R. Ayyagari, "Computational complexity of Kharitonov's robust stability test," International Journal of Control Science and Engineering, vol. 3, no. 3, pp. 81-85, 2013.

[100] C. B. Soh, C. S. Berger, and K. P. Dabke, "On the stability properties of polynomials with perturbed coefficients," IEEE Transactions on Automatic Control, vol. 30, no. 10, pp. 1033-1036, 1985.

[101] Y. Z. Tsypkin and B. T. Polyak, "Frequency domain criteria for $l_{p}$-robust stability of continuos linear systems," IEEE Transactions on Automatic Control, vol. 36, no. 12, pp. 1464-1469, 1991.

[102] A. C. Bartlett, C. V. Hollot, and H. Lin, "Root locations of an entire polytope of polynomials: it suffices to check the edges," Mathematics of Control, Signals, and Systems, vol. 1, no. 1, pp. 61-71, 1988.

[103] F. Kelly, "Mathematical modelling of the Internet," in Mathematics Unlimite-2001 and Beyond, Springer, Berlin, Germany, 2001.

[104] R. M. Murray, Control in an Information Rich World: Report of the Panel on Future Directions in Control, Dynamics, and Systems, SIAM, Minneapolis, MN, USA, 2003.

[105] R. M. Murray, K. J. Astrom, S. P. Boyd, R. W. Brockett, and G. Stein, "Future directions in control in an information-rich world," IEEE Control Systems Magazine, vol. 23, no. 2, pp. 20-33, 2003.

[106] R. J. Anderson and M. W. Spong, "Bilateral control of teleoperators with time delay," IEEE Transactions on Automatic Control, vol. 34, no. 5, pp. 494-501, 1989.

[107] J. E. Speich and J. Rosen, "Medical robotics," Encyclopedia of Biomaterials and Biomedical Engineering, vol. 983, p. 993, 2004.

[108] J. F. M. Rubio, B. d. M. Cuéllar, and S. Olivier, "Control of delayed recycling systems with an unstable pole at forward path," in Proceedings of the 2012 American Control Conference (ACC), pp. 5658-5663, Montreal, Canada, 2012.

[109] K. Gopalsamy, Stability and Oscillations in Delay Differential Equations of Population Dynamics, Springer Science \& Business Media, Berlin, Germany, 2013.

[110] S.-I. Niculescu, C.-I. Morărescu, W. Michiels, and K. Gu, "Geometric ideas in the stability analysis of delay models in biosciences," in Biology and Control Theory: Current Challenges, Springer, Berlin, Germany, 2007.

[111] R. Villafuerte-Segura, E. Alvarado-Santos, and B. A. ItzáOrtiz, "Conditions for stable equilibrium in Cournot duopoly models with tax evasion and time delay," Chaos: An Interdisciplinary Journal of Nonlinear Science, vol. 30, no. 1, Article ID 013142, 2020.

[112] B. A. Itzá-Ortiz, R. Villafuerte-Segura, and E. AlvaradoSantos, "Delay-dependent and delay-independent stability of Cournot duopoly model with tax evasion and time-delay," Communications in Nonlinear Science and Numerical Simulation, 2020.

[113] A. Ramirez, E. S. Espinoza, L. R. G. Carrillo, S. Mondié, A. García, and R. Lozano, "Stability analysis of a vision-based UAV controller," Journal of Intelligent \& Robotic Systems, vol. 74, no. 1-2, pp. 69-84, 2014.

[114] J. Cheong, S.-I. Niculescu, A. Annaswamy, and M. A. Srinivasan, "Synchronization control for physics- based collaborative virtual environments with shared haptics," Advanced Robotics, vol. 21, no. 9, pp. 1001-1029, 2007.

[115] L. Vite-Hernández, R. Villafuerte-Segura, M. Ramírez-Neria, and G. Ochoa-Ortega, " $\sigma$-stabilization of a flexible joint robotic arm via delayed controllers," Complexity, vol. 2019, pp. 1-12, 2019.

[116] T. Ortega-Montiel, R. Villafuerte-Segura, C. VázquezAguilera, and L. Freidovich, "Proportional retarded controller to stabilize underactuated systems with measurement delays: furuta pendulum case study," Mathematical Problems in Engineering, vol. 2017, Article ID 2505086, 2017.

[117] N. N. Krasovskii, Stability of Motion, Stanford University Press, Palo Alto, CA, USA, 1963.

[118] L. E. Elsgolts, Introduction to the Theory of Differential Equations with Deviating Arguments, Holden-Day, San Francisco, CA, USA, 1966.

[119] S. B. Norkin, Introduction to the Theory and Application of Differential Equations with Deviating Arguments, Academic Press, Cambridge, MA, USA, 1973.

[120] W. Hahn, Stability of Motion, Springer, Berlin, Germany, 1967.

[121] Vladimir Borisovich Kolmanovskii and Valerij Romanovič Nosov, Stability of Functional Differential Equations, Elsevier, Amsterdam, Netherlands, 1986.

[122] J. K. Hale and S. M. V. Lunel, Introduction to Functional Differential Equations, Springer Science \& Business Media, Berlin, Germany, 1993.

[123] V. Kharitonov, Time-delay Systems: Lyapunov Functionals and Matrices, Springer Science \& Business Media, Berlin, Germany, 2012.

[124] M. Wu, Y. He, and J.-H. She, Stability Analysis and Robust Control of Time-Delay Systems, Springer, Berlin, Germany, 2010.

[125] M. Lakshmanan and D. Vijayan Senthilkumar, Dynamics of Nonlinear Time-Delay Systems, Springer Science \& Business Media, Berlin, Germany, 2011.

[126] C. Hua, L. Zhang, and X. Guan, Robust Control for Nonlinear Time-Delay Systems, Springer, Berlin, Germany, 2018.

[127] C. Sowmiya, R. Raja, J. Cao, and G. Rajchakit, "Impulsive discrete-time bam neural networks with random parameter uncertainties and time-varying leakage delays: an asymptotic stability analysis," Nonlinear Dynamics, vol. 91, no. 4, pp. 2571-2592, 2018.

[128] B. D. Hassard, B. D. Hassard, N. D. Kazarinoff, Y.-H. Wan, and Y. W. Wan, Theory and Applications of Hopf Bifurcation, CUP Archive, Cambridge, England, UK, 1981.

[129] M. Krstic, P. V. Kokotovic, and I. Kanellakopoulos, Nonlinear and Adaptive Control Design, John Wiley \& Sons, Hoboken, NJ, USA, 1995.

[130] H. K. Khalil and J. W. Grizzle, Nonlinear Systems, PrenticeHall, Upper Saddle River, NJ, USA, 2002.

[131] E. Fridman, A. Pila, and U. Shaked, "Regional stabilization and $H_{\infty}$ control of time-delay systems with saturating actuators," International Journal of Robust and Nonlinear Control, vol. 13, no. 9, pp. 885-907, 2003.

[132] D. Melchor-Aguilar and S.-I. Niculescu, "On computing estimates of the attraction region for a class of nonlinear time-delay systems," IFAC Proceedings Volumes, vol. 39, no. 9, pp. 626-631, 2006.

[133] B. Ding and B. Huang, "Constrained robust model predictive control for time-delay systems with polytopic description," International Journal of Control, vol. 80, no. 4, pp. 509-522, 2007. 
[134] R. Villafuerte, S. Mondié, and S.-I. Niculescu, "Stability analysis and estimate of the region of attraction of a human respiratory model," IMA Journal of Mathematical Control and information, vol. 27, no. 3, pp. 309-327, 2010.

[135] R. Sepulchre, M. Jankovic, and P. V. Kokotovic, Constructive Nonlinear Control, Springer Science \& Business Media, Berlin, Germany, 2012.

[136] S. Sastry, Nonlinear Systems: Analysis, Stability, and Control, Springer Science \& Business Media, Berlin, Germany, 2013.

[137] I. Alberto, Nonlinear Control Systems Design 1989: Selected Papers from the IFAC Symposium, Capri, Italy, 14-16 June 1989, Elsevier, Amsterdam, Netherlands, 2014.

[138] L. S. Pontryagin, "O nuliakh nekotorykh elementarnykh transtsendentnykh funktsii," Izvestiya Akademii Nauk SSSR, vol. 6, no. 1, pp. 115-134, 1942.

[139] L. S. Pontryagin, "On the zeros of some elementary transcendental functions," American Mathematical Society Translations, vol. 2, no. 1, pp. 95-110, 1955.

[140] E. M. Wright, "The non-linear difference-differential equation," The Quarterly Journal of Mathematics, vol. 17, no. 1, pp. 245-252, 1946.

[141] E. M. Wright, "The stability of solutions of non-linear difference-differential equations," Proceedings of the Royal Society of Edinburgh. Section A. Mathematical and Physical Sciences, vol. 63, no. 1, pp. 18-26, 1950.

[142] R. Bellman, "On the boundedness of solutions of nonlinear differential and difference equations," Transactions of the American Mathematical Society, vol. 62, no. 3, p. 357, 1947.

[143] R. E. Bellman and J. M. Danskin, "A survey of the mathematical theory of time-lag, retarded control, and hereditary processes," Technical report, Rand Corporation, Santa Monica, CA, USA, 1954.

[144] K. L. Cooke, "The asymptotic behavior of the solutions of linear and nonlinear differential-difference equations," Transactions of the American Mathematical Society, vol. 75, no. 1, p. $80,1953$.

[145] B. S. Razumikhin, "Stability of delay systems," Prikl. Mat. Mekh, vol. 20, no. 4, pp. 500-512, 1956.

[146] N. N. Krasovskii, "On the application of the second method of lyapunov for equations with time delays," Prikl. Mat. Mekh, vol. 20, no. 3, pp. 315-327, 1956.

[147] Z. Lendek, T. M. Guerra, R. Babuska, and B. De Schutter, Stability Analysis and Nonlinear Observer Design Using Takagi-Sugeno Fuzzy Models, Springer, Berlin, Germany, 2011.

[148] B. d'Andrea Novel, G. Bastin, and G. Campion, "Dynamic feedback linearization of nonholonomic wheeled mobile robots," in Proceedings of the 1992 IEEE International Conference on Robotics and Automation, pp. 2527-2532, Glasgow, Scotland, 1992.

[149] L. Hunt, R. Renjeng Su, and G. Meyer, "Global transformations of nonlinear systems," IEEE Transactions on Automatic Control, vol. 28, no. 1, pp. 24-31, 1983.

[150] H. Kokame and T. Mori, "Stability preserving transition from derivative feedback to its difference counterparts," in Proceedings of the 15th Triennal IFAC World Congress, Barcelona, Spain, 2002.

[151] M. S. Mahmoud and A. Ismail, "Passivity and passification of time-delay systems," Journal of Mathematical Analysis and Applications, vol. 292, no. 1, pp. 247-258, 2004.

[152] C. Li, H. Zhang, and X. Liao, "Passivity and passification of fuzzy systems with time delays," Computers \& Mathematics with Applications, vol. 52, no. 6-7, pp. 1067-1078, 2006.
[153] R. Lozano, N. Chopra, and M. W. Spong, "Passivation of force reflecting bilateral teleoperators with time varying delay," in Proceedings of the 8 Mechatronics Forum, Enschede, Netherlands, June 2002.

[154] J. Lian, P. Shi, and Z. Feng, "Passivity and passification for a class of uncertain switched stochastic time-delay systems," IEEE Transactions on Cybernetics, vol. 43, no. 1, pp. 3-13, 2012.

[155] F. Mazenc and P.-A. Bliman, "Backstepping design for time-delay nonlinear systems," IEEE Transactions on Automatic Control, vol. 51, no. 1, pp. 149-154, 2006.

[156] C. Changchun Hua, X. Xinping Guan, and P. Peng Shi, "Robust backstepping control for a class of time delayed systems," IEEE Transactions on Automatic Control, vol. 50, no. 6, pp. 894-899, 2005.

[157] J. Zhou, C. Wen, and W. Wang, “Adaptive backstepping control of uncertain systems with unknown input timedelay," Automatica, vol. 45, no. 6, pp. 1415-1422, 2009.

[158] C. Changchun Hua, P. X. Liu, and X. Xinping Guan, "Backstepping control for nonlinear systems with time delays and applications to chemical reactor systems," IEEE Transactions on Industrial Electronics, vol. 56, no. 9, pp. 3723-3732, 2009.

[159] S. Monaco, D. Normand-Cyrot, and M. Mattioni, "Sampleddata stabilization of nonlinear dynamics with input delays through immersion and invariance," IEEE Transactions on Automatic Control, vol. 62, no. 5, pp. 2561-2567, 2016.

[160] C. Murguia, R. H. B. Fey, and H. Nijmeijer, "Immersion and invariance observers with time-delayed output measurements," Communications in Nonlinear Science and $\mathrm{Nu}$ merical Simulation, vol. 30, no. 1-3, pp. 227-235, 2016.

[161] C. Murguia, R. H. B. Fey, and H. Nijmeijer, "Network synchronization using invariant-manifold-based diffusive dynamic couplings with time-delay," Automatica, vol. 57, pp. 34-44, 2015.

[162] E. Franco, "Immersion and invariance adaptive control for discrete-time systems in strict-feedback form with input delay and disturbances," International Journal of Adaptive Control and Signal Processing, vol. 32, no. 1, pp. 69-82, 2018.

[163] H. Mounier and J. Rudolph, "Flatness-based control of nonlinear delay systems: a chemical reactor example," International Journal of Control, vol. 71, no. 5, pp. 871-890, 1998.

[164] J. Rudolph and J. Winkler, “A generalized flatness concept for nonlinear delay systems: motivation by chemical reactor models with constant or input dependent delays," International Journal of Systems Science, vol. 34, no. 8-9, pp. 529$541,2003$.

[165] H. Mounier and J. Rudolph, "Flatness and quasi-static state feedback in non-linear delay systems," International Journal of Control, vol. 81, no. 3, pp. 445-456, 2008.

[166] K. Gu, J. Chen, and V. L. Kharitonov, Stability of Time-Delay Systems, Springer Science \& Business Media, Berlin, Germany, 2003.

[167] F. Wu and K. M. Grigoriadis, "LPV systems with parameter-varying time delays: analysis and control," Automatica, vol. 37, no. 2, pp. 221-229, 2001.

[168] J.-H. Kim, "Note on stability of linear systems with time-varying delay," Automatica, vol. 47, no. 9, pp. 2118-2121, 2011.

[169] M. Iswarya, R. Raja, G. Rajchakit, J. Cao, J. Alzabut, and C. Huang, "A perspective on graph theory-based stability analysis of impulsive stochastic recurrent neural networks with time-varying delays," Advances in Difference Equations, vol. 2019, no. 1, pp. 1-21, 2019. 
[170] A. Hurwitz, "Ueber die Bedingungen, unter welchen eine Gleichung nur Wurzeln mit negativen reellen Theilen besitzt," Mathematische Annalen, vol. 46, no. 2, pp. 273-284, 1895.

[171] A. Hurwitz, "Uber die bedingungen, unter welchen eine gleichung nur wurzeln mit negativen reellen theilen besitzt, stability theory (ascona, 1995)," International Series of $\mathrm{Nu}$ merical Mathematics, vol. 121, pp. 239-249, 1995.

[172] E. A. Kalinina, "Stability and D-stability of the family of real polynomials," Linear Algebra and its Applications, vol. 438, no. 6, pp. 2635-2650, 2013.

[173] S. Angulo, R. Marquez, and M. Bernal, "Quasi-polynomialbased robust stability of time-delay systems can be less conservative than Lyapunov-Krasovskii approaches," IEEE Transactions on Automatic Control, vol. 65, no. 7, pp. 3164-3169, 2019.

[174] M. Bernal, V. Estrada, and R. Márquez, "Diseno e implementación de sistemas de control basados en estructuras convexas y desigualdades matriciales lineales," Pearson Mexico City, Mexico, 2019.

[175] K. Ogata and Y. Yang, Modern Control Engineering, Prentice-Hall, Upper Saddle River, NJ, USA, 2010.

[176] W. Michiels and S.-I. Niculescu, "Stability and stabilization of time-delay systems: an eigenvalue-based approach," SIAM, Minneapolis, MN, USA, 2007.

[177] L. S. Pontryagin, "On the zeros of some elementary transcendental functions," American Mathematical Society Translations, vol. 2, no. 1, pp. 95-110, 1955.

[178] Y. M. Yesipovich, "On the stability of the solution of a class of differential equations with delayed argument," Prikl. Mat. Mech., vol. 1, no. 1, pp. 601-608, 1951.

[179] C. S. Hsu, "Application of the $\tau$-decomposition method to dynamical systems subjected to retarded follower forces," Journal of Applied Mechanics, vol. 37, no. 1, pp. 258-266, 1970.

[180] J. I. Neimark, "D-decomposition of the space of quasipolynomials (on the stability of linearized distributive systems)," American Mathematical Society Translations, vol. 102, pp. 95-131, 1973.

[181] H. Nyquist, "Regeneration theory," Bell System Technical Journal, vol. 11, no. 1, pp. 126-147, 1932.

[182] M. Satche, "Discussion on ANSOFF: stability of linear oscillating systems with constant time lag," Journal of Applied Mechanics, vol. 16, no. 1, pp. 419-420, 1949.

[183] A. W. Mikhailov, "Methods for harmonic analysis in the automatic control system theory (in Russian)," Automatic Telemekh, vol. 3, pp. 1-6, 1938.

[184] B. Porter, Stability Criteria for Linear Dynamical Systems, Oliver and Boyd, Edinburgh, London, UK, 1967.

[185] R. Farkh, K. Laabidi, and M. Ksouri, "Stabilizing sets of PI/ PID controllers for unstable second order delay system," International Journal of Automation and Computing, vol. 11, no. 2, pp. 210-222, 2014.

[186] H. Wang, J. Liu, F. Yang, and Y. Zhang, "Proportional-integral controller for stabilization of second-order delay processes," International Journal of Control, Automation and Systems, vol. 12, no. 6, pp. 1197-1206, 2014.

[187] A. C. Bartlett, C. V. Hollot, and H. Lin, "Root locations of an entire polytope of polynomials: it suffices to check the edges," Mathematics of Control, Signals, and Systems, vol. 1, no. 1, pp. 61-71, 1988.

[188] E. Schwengeler, Geometrisches über die Verteilung der Nullstellen spezieller ganzer Funktionen (Exponentialsummen), $\mathrm{PhD}$ thesis, ETH Zurich, Zürich, Switzerland, 1925.
[189] J. G. ZieglerB. Nathaniel et al., "Optimum settings for automatic controllers," Transactions of ASME, vol. 64, no. 11, 1942.

[190] M. Stojic and D. Siljak, "Generalization of Hurwitz, Nyquist, and Mikhailov stability criteria," IEEE Transactions on Automatic Control, vol. 10, no. 3, pp. 250-254, 1965.

[191] V. A. Oliveira, C. M. Marcelo, and L. Cossi, "Stabilizing a class of time delay systems using the hermite-biehler theorem," Linear Algebra and its Applications, vol. 369, pp. 203-216, 2003.

[192] G. Stépán, Retarded Dynamical Systems: Stability and Characteristic Functions, Longman Scientific \& Technical, UK, 1989.

[193] M.-T. Ho, A. Datta, and S. P. Bhattacharyya, "Generalizations of the hermite-biehler theorem," Linear Algebra and its Applications, vol. 302, pp. 135-153, 1999.

[194] G. J. Silva, A. Datta, and S. P. Bhattacharyya, PID Controllers for Time-Delay Systems, Springer Science \& Business Media, Berlin, Germany, 2007.

[195] K. Gu, "A review of some subtleties of practical relevance for time-delay systems of neutral type," ISRN Applied Mathematics, vol. 2012, Article ID 725783, 2012.

[196] V. L. Kharitonov and A. P. Zhabko, "Robust stability of timedelay systems," IEEE Transactions on Automatic Control, vol. 39, no. 12, pp. 2388-2397, 1994.

[197] M. Fu, A. W. Olbrot, and M. P. Polis, "Robust stability for time-delay systems: the edge theorem and graphical tests," IEEE Transactions on Automatic Control, vol. 34, no. 8, pp. 813-820, 1989.

[198] V. L. Kharitonov, J. A. Torres-Muñoz, and M. B. OrtizMoctezuma, "Polytopic families of quasi-polynomials: vertex-type stability conditions," IEEE Transactions on Circuits and Systems I: Fundamental Theory and Applications, vol. 50, no. 11, pp. 1413-1420, 2003.

[199] S. Mondie, J. Santos, and V. L. Kharitonov, "Robust stability of quasi-polynomials and the finite inclusions theorem," IEEE Transactions on Automatic Control, vol. 50, no. 11, pp. 1826-1831, 2005.

[200] G. Stein, G. Hartmann, and R. Hendrick, "Adaptive control laws for f-8 flight test," IEEE Transactions on Automatic Control, vol. 22, no. 5, pp. 758-767, 1977.

[201] J. S. Shamma and M. Athans, "Guaranteed properties of gain scheduled control for linear parameter-varying plants," Automatica, vol. 27, no. 3, pp. 559-564, 1991.

[202] K. Walton and J. E. Marshall, "Direct method for TDS stability analysis," IEE Proceedings D Control Theory and Applications, vol. 134, no. 2, pp. 101-107, 1987.

[203] J. M. Otto, "Closed control of loop with dead time," Chemical Engineering Progress, vol. 53, pp. 217-219, 1957.

[204] Z. Rekasius and J. Gibson, "Stability analysis of nonlinear control systems by the second method of Liapunov," IRE Transactions on Automatic Control, vol. 7, no. 1, pp. 3-15, 1962.

[205] O. Perron, Die lehre von den kettenbriichen ii. Aufl., Teubner, Stuttgart (1954, 1957), 1957.

[206] R. Sipahi, H. Fazelinia, and N. Olgac, "Generalization of cluster treatment of characteristic roots for robust stability of multiple time-delayed systems," International Journal of Robust and Nonlinear Control, vol. 18, no. 14, pp. 1430-1449, 2008.

[207] J. Lam, "Model reduction of delay systems using Pade approximants," International Journal of Control, vol. 57, no. 2, pp. 377-391, 1993. 
[208] A. V. Egorov, C. Cuvas, and S. Mondié, "Necessary and sufficient stability conditions for linear systems with pointwise and distributed delays," Automatica, vol. 80, pp. 218-224, 2017.

[209] I. V. Medvedeva and A. P. Zhabko, "Synthesis of Razumikhin and Lyapunov-Krasovskii approaches to stability analysis of time-delay systems," Automatica, vol. 51, pp. 372-377, 2015.

[210] M. J. Park, O. M. Kwon, J. H. Park, and S. M. Lee, “A new augmented Lyapunov-Krasovskii functional approach for stability of linear systems with time-varying delays," Applied Mathematics and Computation, vol. 217, no. 17, pp. 71977209, 2011.

[211] P.-L. Liu, "Exponential stability for linear time-delay systems with delay dependence," Journal of the Franklin Institute, vol. 340, no. 6-7, pp. 481-488, 2003.

[212] X. Li and C. E. De Souza, "Criteria for robust stability and stabilization of uncertain linear systems with state delay," Automatica, vol. 33, no. 9, pp. 1657-1662, 1997.

[213] K. Gu, "Discretized LMI set in the stability problem of linear uncertain time-delay systems," International Journal of Control, vol. 68, no. 4, pp. 923-934, 1997.

[214] L. Dugard and E. I. Verriest, Stability and Control of TimeDelay Systems, Springer, Berlin, Germany, 1998.

[215] P. G. Park, "A delay-dependent stability criterion for systems with uncertain time-invariant delays," IEEE Transactions on Automatic Control, vol. 44, no. 4, pp. 876-877, 1999.

[216] K. Gu, "An integral inequality in the stability problem of time-delay systems," in Proceedings of the 39th IEEE Conference on Decision and Control, pp. 2805-2810, Sydney, Australia, December 2000.

[217] E. Fridman, "New Lyapunov-Krasovskii functionals for stability of linear retarded and neutral type systems," Systems \& Control Letters, vol. 43, no. 4, pp. 309-319, 2001.

[218] Y. S. Moon, P. Park, W. H. Kwon, and Y. S. Lee, "Delaydependent robust stabilization of uncertain state-delayed systems," International Journal of Control, vol. 74, no. 14, pp. 1447-1455, 2010.

[219] E. Fridman and U. Shaked, "A descriptor system approach to $\mathrm{H} /$ sub $\infty /$ control of linear time-delay systems," IEEE Transactions on Automatic Control, vol. 47, no. 2, pp. 253270, 2002.

[220] Q.-L. Han, "Robust stability of uncertain delay-differential systems of neutral type," Automatica, vol. 38, no. 4, pp. 719-723, 2002.

[221] E. Fridman and U. Shaked, "An improved stabilization method for linear time-delay systems," IEEE Transactions on Automatic Control, vol. 47, no. 11, pp. 1931-1937, 2002.

[222] X. Zhang, P. Tsiotras, and C. Knospe, "Stability analysis of LPV time-delayed systems," International Journal of Control, vol. 75, no. 7, pp. 538-558, 2002.

[223] E. Fridman and U. Shaked, "Delay-dependent stability and $H_{\infty}$ control: constant and time-varying delays," International Journal of Control, vol. 76, no. 1, pp. 48-60, 2003.

[224] V. L. Kharitonov and D. Hinrichsen, "Exponential estimates for time delay systems," Systems \& Control Letters, vol. 53, no. 5, pp. 395-405, 2004.

[225] E. Fridman, A. Seuret, and J.-P. Richard, "Robust sampleddata stabilization of linear systems: an input delay approach," Automatica, vol. 40, no. 8, pp. 1441-1446, 2004.

[226] M. Wu, Y. He, and J.-H. She, "New delay-dependent stability criteria and stabilizing method for neutral systems," IEEE Transactions on Automatic Control, vol. 49, no. 12, pp. 2266-2271, 2004.
[227] Y. He, M. Wu, J.-H. She, and G.-P. Liu, "Parameter-dependent Lyapunov functional for stability of time-delay systems with polytopic-type uncertainties," IEEE Transactions on Automatic Control, vol. 49, no. 5, pp. 828-832, 2004.

[228] S. Mondie and V. L. Kharitonov, "Exponential estimates for retarded time-delay systems: an LMI approach," IEEE Transactions on Automatic Control, vol. 50, no. 2, pp. 268273, 2005.

[229] S. Shengyuan, J. Xu, and J. Lam, "Improved delay-dependent stability criteria for time-delay systems," IEEE Transactions on Automatic Control, vol. 50, no. 3, pp. 384-387, 2005.

[230] S. Xu, J. Lam, and M. Zhong, "New exponential estimates for time-delay systems," IEEE Transactions on Automatic Control, vol. 51, no. 9, pp. 1501-1505, 2006.

[231] S. Xu and J. Lam, Robust Control and Filtering of Singular Systems, Springer, Berlin, Germany, 2006.

[232] X. Jiang and Q.-L. Han, "Delay-dependent robust stability for uncertain linear systems with interval time-varying delay," Automatica, vol. 42, no. 6, pp. 1059-1065, 2006.

[233] Y. He, Q.-G. Wang, C. Lin, and M. Wu, "Delay-range-dependent stability for systems with time-varying delay," Automatica, vol. 43, no. 2, pp. 371-376, 2007.

[234] H. Shao, "New delay-dependent stability criteria for systems with interval delay," Automatica, vol. 45, no. 3, pp. 744-749, 2009.

[235] J. Sun, G. P. Liu, J. Chen, and D. Rees, "Improved delayrange-dependent stability criteria for linear systems with time-varying delays," Automatica, vol. 46, no. 2, pp. 466-470, 2010.

[236] H.-B. Zeng, Y. He, M. Wu, and J. She, "Free-matrix-based integral inequality for stability analysis of systems with timevarying delay," IEEE Transactions on Automatic Control, vol. 60, no. 10, pp. 2768-2772, 2015.

[237] R. S. Kumar, G. Sugumaran, R. Raja, Q. Zhu, and U. K. Raja, "New stability criterion of neural networks with leakage delays and impulses: a piecewise delay method," Cognitive Neurodynamics, vol. 10, no. 1, pp. 85-98, 2016.

[238] S. Pandiselvi, R. Raja, J. Cao, X. Li, and G. Rajchakit, "Impulsive discrete-time GRNS with probabilistic time delays, distributed and leakage delays: an asymptotic stability issue," IMA Journal of Mathematical Control and Information, vol. 36, no. 1, pp. 79-100, 2019.

[239] V. Kharitonov, "Robust stability analysis of time delay systems: a survey," Annual Reviews in Control, vol. 23, no. 1, pp. 185-196, 1999.

[240] K. Gu and S.-I. Niculescu, "Survey on recent results in the stability and control of time-delay systems," Journal of Dynamic Systems, Measurement, and Control, vol. 125, no. 2, pp. 158-165, 2003.

[241] J.-P. Richard, "Time-delay systems: an overview of some recent advances and open problems," Automatica, vol. 39, no. 10, pp. 1667-1694, 2003.

[242] I. M. Repin, "Quadratic Liapunov functionals for systems with delay," Journal of Applied Mathematics and Mechanics, vol. 29, no. 3, pp. 669-672, 1965.

[243] R. Datko, "An algorithm for computing Liapunov functionals for some differential-difference equations," in Ordinary Differential Equations, Elsevier, Amsterdam, Netherlands, 1972.

[244] E. F. Infante and W. B. Castelan, A Liapunov Functional for a Matrix Difference-Differential Equation, Technical report, Brown University, Providence, RI, USA, 1977. 
[245] H. Wenzhang, “Generalization of Liapunov's theorem in a linear delay system," Journal of Mathematical Analysis and Applications, vol. 142, no. 1, pp. 83-94, 1989.

[246] V. L. Kharitonov and A. P. Zhabko, "Lyapunov-Krasovskii approach to robust stability of time delay systems," IFAC Proceedings Volumes, vol. 34, no. 13, pp. 477-481, 2001.

[247] V. L. Kharitonov and A. P. Zhabko, "Lyapunov-Krasovskii approach to the robust stability analysis of time-delay systems," Automatica, vol. 39, no. 1, pp. 15-20, 2003.

[248] H. Garcia-Lozano and V. L. Kharitonov, "Lyapunov matrices for time delay system with commensurate delays," IFAC Proceedings Volumes, vol. 37, no. 21, pp. 91-95, 2004.

[249] H. Garcia-Lozano and V. L. Kharitonov, "Numerical computation of time delay Lyapunov matrices," IFAC Proceedings Volumes, vol. 39, no. 10, pp. 60-65, 2006.

[250] R. Villafuerte, S. Mondie, and O. Santos, "On the computation of estimates for time delay systems with Lyapunov-Krasovskii functionals of complete type," in Proceedings of the 2006 3rd International Conference on Electrical and Electronics Engineering, pp. 1-4, Veracruz, Mexico, September 2006.

[251] B. M. Ochoa and S. Mondie, "Approximations of LyapunovKrasovskii functionals of complete type with given cross terms in the derivative for the stability of time delay systems," in Proceedings of the 2007 46th IEEE Conference on Decision and Control, pp. 2071-2076, New Orleans, LO, USA, December 2007.

[252] V. L. Kharitonov, "Lyapunov matrices for a class of neutral type time delay systems," International Journal of Control, vol. 81, no. 6, pp. 883-893, 2008.

[253] J. E. Velázquez and V. L. Kharitonov, "Lyapunov-Krasovskii functionals for scalar neutral type time delay equations," Systems \& Control Letters, vol. 58, no. 1, pp. 17-25, 2009.

[254] A. V. Egorov and V. L. Kharitonov, "Approximation of delay Lyapunov matrices," International Journal of Control, vol. 91, no. 11, pp. 2588-2596, 2018.

[255] S. Mondie, V. L. Kharitonov, and O. Santos, "Complete type Lyapunov-Krasovskii functionals with a given cross term in the time derivative," in Proceedings of the 44th IEEE Conference on Decision and Control, pp. 5060-5064, Seville, Spain, 2005.

[256] O. Santos, S. Mondie, and V. L. Kharitonov, "Robust stability conditions for systems with distributed delays," in Proceedings of the 45th IEEE Conference on Decision and Control, pp. 217-222, San Diego, CA, USA, December 2006.

[257] E. Fridman, "Stability of systems with uncertain delays: a new "complete" Lyapunov-Krasovskii functional," IEEE Transactions on Automatic Control, vol. 51, no. 5, pp. 885890, 2006.

[258] E. Fridman and S.-I. Niculescu, "On complete LyapunovKrasovskii functional techniques for uncertain systems with fast-varying delays," International Journal of Robust and Nonlinear Control, vol. 18, no. 3, pp. 364-374, 2008.

[259] O. Santos, S. Mondié, and V. L. Kharitonov, "Linear quadratic suboptimal control for time delays systems," IFAC Proceedings Volumes, vol. 39, no. 10, pp. 96-101, 2006.

[260] V. L. Kharitonov, "Predictor based stabilization of neutral type systems with input delay," Automatica, vol. 52, pp. 125-134, 2015.

[261] V. L. Kharitonov, "Prediction-based control for systems with state and several input delays," Automatica, vol. 79, pp. 11-16, 2017.

[262] A. N. Aliseyko and V. L. Kharitonov, "Lyapunov-Krasovskii functionals for linear systems with input delay," IFACPapersOnLine, vol. 52, no. 18, pp. 19-24, 2019.
[263] L. Juárez, S. Mondié, and V. L. Kharitonov, "Dynamic predictor for systems with state and input delay: a timedomain robust stability analysis," International Journal of Robust and Nonlinear Control, vol. 30, no. 6, pp. 2204-2218, 2020.

[264] L. A. Castañeda, A. Luviano-Juárez, G. Ochoa-Ortega, and I. Chairez, "Tracking control of uncertain time delay systems: an ADRC approach," Control Engineering Practice, vol. 78, pp. 97-104, 2018.

[265] M. A. Gomez, W. Michiels, and S. Mondié, "Design of delaybased output-feedback controllers optimizing a quadratic cost function via the delay Lyapunov matrix," Automatica, vol. 107, pp. 146-153, 2019.

[266] M. A. Gomez, A. V. Egorov, and S. Mondié, "Lyapunov matrix based necessary and sufficient stability condition by finite number of mathematical operations for retarded type systems," Automatica, vol. 108, Article ID 108475, 2019.

[267] M. A. Gomez, A. V. Egorov, S. Mondié, and A. P. Zhabko, "Computation of the Lyapunov matrix for periodic timedelay systems and its application to robust stability analysis," Systems \& Control Letters, vol. 132, Article ID 104501, 2019.

[268] A. Bellen and M. Zennaro, Numerical Methods for Delay Differential Equations, Oxford University Press, Oxford, UK, 2013.

[269] K. Engelborghs, T. Luzyanina, and D. Roose, "Numerical bifurcation analysis of delay differential equations using DDE-Biftool," ACM Transactions on Mathematical Software, vol. 28, no. 1, pp. 1-21, 2002.

[270] E. Hairer, S. P. Nørsett, and G. Wanner, "Solving ordinary differential equations $i$ Nonstiff Problems," Springer Series in Computational Mathematics, vol. 8, Berlin, Germany, 1993.

[271] D. Breda, S. Maset, and R. Vermiglio, Stability of Linear Delay Differential Equations: A Numerical Approach with MATLAB, Springer, Berlin, Germany, 2014.

[272] J. Louisell, "Numerics of the stability exponent and eigenvalue abscissas of a matrix delay system," in Stability and Control of Time-Delay Systems, Springer, Berlin, Germany, 1998.

[273] J. Louisell, "Stability exponent and eigenvalue abscissas by way of the imaginary axis eigenvalues," in Advances in TimeDelay Systems, Springer, Berlin, Germany, 2004.

[274] J. Louisell, "A matrix method for determining the imaginary axis eigenvalues of a delay system," IEEE Transactions on Automatic Control, vol. 46, no. 12, pp. 2008-2012, 2001.

[275] G. Ochoa, S. Mondié, and V. L. Kharitonov, "Time delay systems with distributed delays: critical values," IFAC Proceedings Volumes, vol. 42, no. 14, pp. 272-277, 2009.

[276] G. Ochoa, V. L. Kharitonov, and S. Mondié, "Critical frequencies and parameters for linear delay systems: a Lyapunov matrix approach," Systems \& Control Letters, vol. 62, no. 9, pp. 781-790, 2013.

[277] N. Olgac and R. Sipahi, "The cluster treatment of characteristic roots and the neutral type time-delayed systems," Journal of Dynamic Systems, Measurement, and Control, vol. 127, no. 1, pp. 88-97, 2004.

[278] R. Sipahi and N. Olgac, "A novel stability study on multiple time-delay systems (MTDS) using the root clustering paradigm," in Proceedings of the 2004 American Control Conference, pp. 5422-5427, Boston, MA, USA, June 2004.

[279] R. Sipahi and N. Olgac, "A unique methodology for the stability robustness of multiple time delay systems," Systems \& Control Letters, vol. 55, no. 10, pp. 819-825, 2006. 
[280] N. Olgac and R. Sipahi, "A practical method for analyzing the stability of neutral type LTI-time delayed systems," Automatica, vol. 40, no. 5, pp. 847-853, 2004.

[281] R. Sipahi and N. Olgac, "Complete stability robustness of third-order LTI multiple time-delay systems," Automatica, vol. 41, no. 8, pp. 1413-1422, 2005.

[282] R. Sipahi, Cluster treatment of characteristic roots, CTCR, a unique methodology for the complete robustness analysis of linear time invariant multiple time delay systems against delay uncertainties, $\mathrm{PhD}$ thesis, University of Connecticut, Mansfield, CT, USA, 2005.

[283] E. Jarlebring, W. Michiels, and K. Meerbergen, "A linear eigenvalue algorithm for the nonlinear eigenvalue problem," Numerische Mathematik, vol. 122, no. 1, pp. 169-195, 2012.

[284] E. Jarlebring, K. Meerbergen, and W. Michiels, "Computing a partial Schur factorization of nonlinear eigenvalue problems using the infinite arnoldi method," SIAM Journal on Matrix Analysis and Applications, vol. 35, no. 2, pp. 411-436, 2014.

[285] R. Van Beeumen, E. Jarlebring, and W. Michiels, “A rankexploiting infinite arnoldi algorithm for nonlinear eigenvalue problems," Numerical Linear Algebra with Applications, vol. 23, no. 4, pp. 607-628, 2016.

[286] W. Michiels, I. Boussaada, and S.-I. Niculescu, "An explicit formula for the splitting of multiple eigenvalues for nonlinear eigenvalue problems and connections with the linearization for the delay eigenvalue problem," SIAM Journal on Matrix Analysis and Applications, vol. 38, no. 2, pp. 599-620, 2017.

[287] W. Michiels and N. Guglielmi, "An iterative method for computing the pseudospectral abscissa for a class of nonlinear eigenvalue problems," SIAM Journal on Scientific Computing, vol. 34, no. 4, pp. A2366-A2393, 2012.

[288] C. Abdallah, D. Peter, J. Benites-Read, and R. Byrne, "Delayed positive feedback can stabilize oscillatory systems," in Proceedings of the 1993 American Control Conference, pp. 3106-3107, San Francisco, CA, USA, June 1993.

[289] W. Michiels and S.-I. Niculescu, "Stability, control, and computation for time-delay systems: an eigenvalue-based approach," SIAM, vol. 27, 2014.

[290] K. Pyragas, "Continuous control of chaos by self-controlling feedback," Physics Letters A, vol. 170, no. 6, pp. 421-428, 1992.

[291] H. Chang, K. J. Åström, and K. Weng, "Refinements of the Ziegler-Nichols tuning formula," in Proceedings of the IEEE $D$ (Control Theory and Applications), pp. 111-118, 1991.

[292] M. E. Bleich and E. S. Joshua, "Stability of periodic orbits controlled by time-delay feedback," Physics Letters A, vol. 210, no. 1-2, pp. 87-94, 1996.

[293] M. de Sousa Vieira and A. J. Lichtenberg, "Controlling chaos using nonlinear feedback with delay," Physical Review E, vol. 54, no. 2, pp. 1200-1207, 1996.

[294] W. Just, T. Bernard, M. Ostheimer, E. Reibold, and H. Benner, "Mechanism of time-delayed feedback control," Physical Review Letters, vol. 78, no. 2, pp. 203-206, 1997.

[295] H. Suh and Z. Bien, "Use of time-delay actions in the controller design," IEEE Transactions on Automatic Control, vol. 25, no. 3, pp. 600-603, 1980.

[296] G. M. Swisher and S. Tenqchen, "Design of proportionalminus-delay action feedback controllers for second-and third-order systems," in Proceedings of the 1988 American Control Conference, pp. 254-260, Atlanta, GA, USA, 1988.

[297] Y. J. Huang, T. C. Kuo, and H. K. Lee, "Fuzzy-PD controller design with stability equations for electro-hydraulic servo systems," in Proceedings of the 2007 International Conference on Control, Automation and Systems, pp. 2407-2410, Seoul, Republic of Korea, 2007.

[298] C.-Y. LinY.-P. Chiu et al., "Catching algorithm for $2 \mathrm{~d}$ robot manipulator using PD controller," in Proceedings of the 2009 ICCAS-SICE, pp. 46-50, Fukuoka, Japan, 2009.

[299] D. Simhachalam, C. Dey, and R. K. Mudi, "An auto-tuning PD controller for DC servo position control system," in Proceedings of the 2012 2nd International Conference on Power, Control and Embedded Systems, pp. 1-6, Allahabad, UP, India, December 2012.

[300] R. Kelly, R. Ortega, A. Ailon, and A. Loria, "Global regulation of flexible joint robots using approximate differentiation," IEEE Transactions on Automatic Control, vol. 39, no. 6, pp. 1222-1224, 1994.

[301] S. Mondié, R. Villafuerte, and R. Garrido, "Tuning and noise attenuation of a second order system using proportional retarded control," IFAC Proceedings Volumes, vol. 44, no. 1, pp. 10337-10342, 2011.

[302] R. Villafuerte, S. Mondié, and R. Garrido, "Tuning of proportional retarded controllers: theory and experiments," IEEE Transactions on Control Systems Technology, vol. 21, no. 3, pp. 983-990, 2012.

[303] J.-E. Hernández-Díez, C.-F. Méndez-Barrios, S. Mondié, S.-I. Niculescu, and E. J. González-Galván, "Proportionaldelayed controllers design for LTI-systems: a geometric approach," International Journal of Control, vol. 91, no. 4, pp. 907-925, 2018.

[304] A. Ramírez, R. Garrido, and S. Mondié, "Integral retarded velocity control of DC servomotors," IFAC Proceedings Volumes, vol. 46, no. 3, pp. 558-563, 2013.

[305] A. Ramírez, R. Sipahi, S. Mondié, and R. Garrido, "Design of maximum decay rate for SISO systems with delayed output feedback using elimination theory," IFAC-PapersOnLine, vol. 48, no. 12, pp. 221-226, 2015.

[306] A. Ramirez, S. Mondié, R. Garrido, and R. Sipahi, "Design of proportional-integral-retarded (PIR) controllers for secondorder LTI systems," IEEE Transactions on Automatic Control, vol. 61, no. 6, pp. 1688-1693, 2016.

[307] R. Villafuerte-Segura, F. Medina-Dorantes, L. ViteHernández, and B. Aguirre-Hernández, "Tuning of a timedelayed controller for a general class of second-order linear time invariant systems with dead-time," IET Control Theory \& Applications, vol. 13, no. 3, pp. 451-457, 2018.

[308] G. Ochoa-Ortega, R. Villafuerte-Segura, M. Ramírez-Neria, and L. Vite-Hernández, $\sigma$-stabilization of a flexible joint robotic arm via delayed controllers," Complexity, vol. 2019, Article ID 7289689, 12 pages, 2019.

[309] D. Melchor-Aguilar and S.-I. Niculescu, "Computing nonfragile pi controllers for delay models of TCP/AQM networks," International Journal of Control, vol. 82, no. 12, pp. 2249-2259, 2009.

[310] Y. Yu, H. Dong, Z. Wang, W. Ren, and F. E. Alsaadi, "Design of non-fragile state estimators for discrete time-delayed neural networks with parameter uncertainties," Neurocomputing, vol. 182, pp. 18-24, 2016.

[311] J.-E. Hernandez-Diez, C.-F. Mendez-Barrios, S.-I. Niculescu, E.-J. Gonzalez-Galvan, G. Mejia-Rodriguez, and V. RamirezRivera, "Closed-loop stability analysis of voltage mode buck using a proportional-delayed controller," in Proceedings of the 2017 25th Mediterranean Conference on Control and Automation (MED), pp. 490-495, Valletta, Malta, July 2017.

[312] S. H. Strogatz, "Exploring complex networks," Nature, vol. 410, no. 6825, pp. 268-276, 2001. 
[313] S. Boccaletti, V. Latora, Y. Moreno, M. Chavez, and D.-U. Hwang, "Complex networks: structure and dynamics," Physics Reports, vol. 424, no. 4-5, pp. 175-308, 2006.

[314] R. Barabási and A.-L. Barabási, "Statistical mechanics of complex networks," Reviews of Modern Physics, vol. 74, no. 1, pp. 47-97, 2002.

[315] C. P. Li, W. G. Sun, and J. Kurths, "Synchronization of complex dynamical networks with time delays," Physica A: Statistical Mechanics and its Applications, vol. 361, no. 1, pp. 24-34, 2006.

[316] L. Su, Y. Wei, W. Michiels, E. Steur, and H. Nijmeijer, "Robust partial synchronization of delay-coupled networks," Chaos: An Interdisciplinary Journal of Nonlinear Science, vol. 30, no. 1, Article ID 013126, 2020.

[317] W. Michiels and H. Nijmeijer, "Synchronization of delaycoupled nonlinear oscillators: an approach based on the stability analysis of synchronized equilibria," Chaos: $A n$ interdisciplinary Journal of Nonlinear Science, vol. 19, no. 3, Article ID 033110, 2009.

[318] M. G. Earl and S. H. Strogatz, "Synchronization in oscillator networks with delayed coupling: a stability criterion," Physical Review E, vol. 67, no. 3, Article ID 036204, 2003.

[319] U. Ernst, K. Pawelzik, and T. Geisel, "Delay-induced multistable synchronization of biological oscillators," Physical Review E, vol. 57, no. 2, pp. 2150-2162, 1998.

[320] E. M. Izhikevich, "Weakly pulse-coupled oscillators, fm interactions, synchronization, and oscillatory associative memory," IEEE Transactions on Neural Networks, vol. 10, no. 3, pp. 508-526, 1999.

[321] M. Y. Choi, H. J. Kim, D. Kim, and H. Hong, "Synchronization in a system of globally coupled oscillators with time delay,” Physical Review E, vol. 61, no. 1, pp. 371-381, 2000.

[322] A. Papachristodoulou and J. Ali, "Synchronization in oscillator networks: switching topologies and non-homogeneous delays," in Proceedings of the 44th IEEE Conference on Decision and Control, pp. 5692-5697, Seville, Spain, 2005.

[323] H.-J. Wünsche, S. Bauer, J. Kreissl et al., "Synchronization of delay-coupled oscillators: a study of semiconductor lasers," Physical Review Letters, vol. 94, no. 16, Article ID 163901, 2005.

[324] A. A. Selivanov, Judith Lehnert, T. Dahms, P. Hövel, A. L. Fradkov, and E. Schöll, "Adaptive synchronization in delay-coupled networks of stuart-landau oscillators," Physical Review E, vol. 85, no. 1, Article ID 016201, 2012.

[325] C. Maharajan, R. Raja, J. Cao, G. Rajchakit, Z. Tu, and A. Alsaedi, "LMI-based results on exponential stability of bam-type neural networks with leakage and both timevarying delays: a non-fragile state estimation approach," Applied Mathematics and Computation, vol. 326, pp. 33-55, 2018.

[326] Q. Song, G. Lu, G. Wen, J. Cao, and F. Liu, "Bipartite synchronization and convergence analysis for network of harmonic oscillator systems with signed graph and time delay," IEEE Transactions on Circuits and Systems I: Regular Papers, vol. 66, no. 7, pp. 2723-2734, 2019.

[327] Y. Yang, W. He, Q.-L. Han, and C. Peng, " $H_{\infty}$ synchronization of networked master-slave oscillators with delayed position data: the positive effects of network-induced delays," IEEE Transactions on Cybernetics, vol. 49, no. 12, pp. 4090-4102, 2019.

[328] R. Huan, D. Pu, X. Wang, and X. Wei, "Effects of phase delay on synchronization in a nonlinear micromechanical oscillator," Applied Physics Letters, vol. 114, no. 23, Article ID 233501, 2019.
[329] I. Choi, H. Culbertson, M. R. Miller, A. Olwal, and S. Follmer, "Grabity: a wearable haptic interface for simulating weight and grasping in virtual reality," in Proceedings of the 30th Annual ACM Symposium on User Interface Software and Technology, pp. 119-130, Québec, Canada, October 2017.

[330] Y. Song, S. Guo, X. Yin et al., "Design and performance evaluation of a haptic interface based on MR fluids for endovascular tele-surgery," Microsystem Technologies, vol. 24, no. 2, pp. 909-918, 2018.

[331] S. Yun, S. Park, B. Park, S. Ryu, S. M. Jeong, and K.-U. Kyung, "A soft and transparent Visuo-haptic interface pursuing wearable devices," IEEE Transactions on Industrial Electronics, vol. 67, no. 1, pp. 717-724, 2019.

[332] P. Polygerinos, F. Sebastian, Q. Fu, and M. Santello, "Soft robotic haptic interface with variable stiffness for rehabilitation of sensorimotor hand function," US Patent App. 16/212, 205, 2019.

[333] R. Brent Gillespie, "Haptic interface to virtual environments," in Robotics and Automation Handbook, CRC Press, Boca Raton, FL, USA, 2018.

[334] G. Hernández-Melgarejo, D. A. Flores-Hernández, A. Luviano-Juárez, L. A. Castañeda, I. Chairez, and S. Di Gennaro, "Mechatronic design and implementation of a bicycle virtual reality system," ISA Transactions, vol. 97, pp. 336-351, 2020.

[335] T. Waltemate, I. Senna, F. Hülsmann et al., "The impact of latency on perceptual judgments and motor performance in closed-loop interaction in virtual reality," in Proceedings of the 22nd ACM Conference on Virtual Reality Software and Technology, pp. 27-35, Munich, Germany, November 2016.

[336] M. S. Elbamby, C. Perfecto, M. Bennis, and K. Doppler, "Toward low-latency and ultra-reliable virtual reality," IEEE Network, vol. 32, no. 2, pp. 78-84, 2018.

[337] R. S. Allison, L. R. Harris, Michael Jenkin, U. Jasiobedzka, and J. E. Zacher, "Tolerance of temporal delay in virtual environments," in Proceedings of the 2001 IEEE Virtual Reality, pp. 247-254, Yokohama, Japan, March 2001.

[338] D. Delaney, T. Ward, and S. McLoone, "On consistency and network latency in distributed interactive applications: a survey-part I," Presence: Teleoperators and Virtual Environments, vol. 15, no. 2, pp. 218-234, 2006.

[339] N. Chopra, M. W. Spong, S. Hirche, and M. Buss, "Bilateral teleoperation over the Internet: the time varying delay," in Proceedings of the American Control Conference (ACC), Denver, CO, USA, June 2003.

[340] N. Chopra, P. Berestesky, and M. W. Spong, "Bilateral teleoperation over unreliable communication networks," IEEE Transactions on Control Systems Technology, vol. 16, no. 2, pp. 304-313, 2008.

[341] P. F. Hokayem and M. W. Spong, "Bilateral teleoperation: an historical survey," Automatica, vol. 42, no. 12, pp. 2035-2057, 2006.

[342] D. A. Lawrence, "Stability and transparency in bilateral teleoperation," IEEE Transactions on Robotics and Automation, vol. 9, no. 5, pp. 624-637, 1993.

[343] K. J. Astrom, C. C. Hang, and B. C. Lim, "A new smith predictor for controlling a process with an integrator and long dead-time," IEEE Transactions on Automatic Control, vol. 39, no. 2, pp. 343-345, 1994.

[344] W. Zhang, Y. Sun, and X. Xu, "Two degree-of-freedom Smith predictor for processes with time delay," Automatica, vol. 34, no. 10, pp. 1279-1282, 1998. 
[345] M. Velasco-Villa, B. del Muro-Cuellar, and A. AlvarezAguirre, "Smith-predictor compensator for a delayed omnidirectional mobile robot," in Proceedings of the 2007 Mediterranean Conference on Control \& Automation, pp. 1-6, Athens, Greece, June 2007.

[346] M. Fliess, R. Marquez, and H. Mounier, "An extension of predictive control, PID regulators and Smith predictors to some linear delay systems," International Journal of Control, vol. 75, no. 10, pp. 728-743, 2002.

[347] M. Fliess, R. Marquez, and H. Mounier, "PID-like regulators for a class of linear delay systems," in Proceedings of the 2001 European Control Conference (ECC), pp. 178-183, Porto, Portugal, 2001.

[348] R. Marquez, M. Fliess, and H. Mounier, "A non-conventional robust pi-controller for the smith predictor," in Proceedings of the 40th IEEE Conference on Decision and Control, pp. 2259-2260, Orlando, FL, USA, 2001.

[349] M. Fliess, R. Marquez, and H. Mounier, "On a generalization of PID regulators for delay systems," IFAC Proceedings Volumes, vol. 34, no. 23, pp. 13-18, 2001.

[350] R. Raja, R. Sakthivel, and S. M. Anthoni, "Stability analysis for discrete-time stochastic neural networks with mixed time delays and impulsive effects," Canadian Journal of Physics, vol. 88, no. 12, pp. 885-898, 2010.

[351] M. Ramirez-Neria, H. Sira-Ramírez, A. Luviano-Juarez, and A. Rodríguez-Angeles, "Smith predictor based generalized PI control for a class of input delayed nonlinear mechanical systems," in Proceedings of the 2013 European Control Conference (ECC), pp. 1292-1297, Zurich, Switzerland, July 2013.

[352] H. Sira-Ramírez, A. Luviano-Juárez, M. Ramírez-Neria, and E. W. Zurita-Bustamante, Active Disturbance Rejection Control of Dynamic Systems: A Flatness Based Approach, Butterworth-Heinemann, Oxford, UK, 2017.

[353] P. Escudero-Gomez, H. Sira-Ramirez, and A. RodriguezAngeles, "Inventory trajectory tracking via an demandatestimation using smithâ predictor," Congreso Nacional de Control Automáatico, Monterrey, Nuevo Leóon, Mexico, pp. 409-414, 2017.

[354] S. Chen, W. Xue, Yi Huang, and P. Liu, "On comparison between smith predictor and predictor observer based ADRCS for nonlinear uncertain systems with output delay," in Proceedings of the 2017 American Control Conference (ACC), pp. 5083-5088, Seattle, WA, USA, May 2017.

[355] W. D. Zhang and Y. X. Sun, "Modified smith predictor for controlling integrator/time delay processes," Industrial \& Engineering Chemistry Research, vol. 35, no. 8, pp. 27692772, 1996.

[356] T. Liu, W. D. Zhang, Y. Z. Cai, and D. Y. Gu, "New modified smith predictor scheme for integrating and unstable processes with time delay," IEE Proceedings-Control Theory and Applications, vol. 152, no. 2, pp. 238-246, 2005.

[357] H. U. Tianjian and X. Huang, "Active disturbance rejection control for space master-slave teleoperation," Journal of Spacecraft TT \& C Technology, vol. 2011, no. 6, p. 8, 2011.

[358] T. J. Hu, X. X. Huang, and Q. Tan, "Active disturbance rejection controller for space teleoperation," in Proceedings of the International Conference on Automatic Control and Artificial Intelligence, pp. 334-337, Xiamen, China, March 2012.

[359] A. C. Smith and K. V. Hashtrudi-Zaad, "Neural networkbased teleoperation using smith predictors," in Proceedings of the IEEE International Conference Mechatronics and
Automation, vol. 3, pp. 1654-1659, Niagara Falls, Ontario, Canada, July 2005.

[360] A. C. Smith and K. Hashtrudi-Zaad, "Smith predictor type control architectures for time delayed teleoperation," The International Journal of Robotics Research, vol. 25, no. 8, pp. 797-818, 2006.

[361] G. Niemeyer and J.-J. E. Slotine, "Stable adaptive teleoperation," IEEE Journal of Oceanic Engineering, vol. 16, no. 1, pp. 152-162, 1991.

[362] G. Niemeyer and J.-J. E. Slotine, "Telemanipulation with time delays," The International Journal of Robotics Research, vol. 23, no. 9, pp. 873-890, 2004.

[363] D. Lee and M. W. Spong, "Passive bilateral teleoperation with constant time delay," IEEE Transactions on Robotics, vol. 22, no. 2, pp. 269-281, 2006.

[364] J.-H. Ryu, J. Artigas, and C. Preusche, "A passive bilateral control scheme for a teleoperator with time-varying communication delay," Mechatronics, vol. 20, no. 7, pp. 812-823, 2010.

[365] N. Chopra, M. W. Spong, and R. Lozano, "Synchronization of bilateral teleoperators with time delay," Automatica, vol. 44, no. 8, pp. 2142-2148, 2008.

[366] E. Nuño, L. Basañez, and R. Ortega, "Passivity-based control for bilateral teleoperation: a tutorial," Automatica, vol. 47, no. 3, pp. 485-495, 2011.

[367] E. Nuño, L. Basañez, R. Ortega, and M. W. Spong, "Position tracking for non-linear teleoperators with variable time delay," The International Journal of Robotics Research, vol. 28, no. 7, pp. 895-910, 2009.

[368] M. Franken, S. Stramigioli, S. Misra, C. Secchi, and A. Macchelli, "Bilateral telemanipulation with time delays: a two-layer approach combining passivity and transparency," IEEE Transactions on Robotics, vol. 27, no. 4, pp. 741-756, 2011.

[369] A. F. Villaverde, A. B. Blas, J. Carrasco, and A. B. Torrico, "Reset control for passive bilateral teleoperation," IEEE Transactions on Industrial Electronics, vol. 58, no. 7, pp. 3037-3045, 2010.

[370] J. Rebelo and A. Schiele, "Time domain passivity controller for 4-channel time-delay bilateral teleoperation," IEEE Transactions on Haptics, vol. 8, no. 1, pp. 79-89, 2014.

[371] D. Sun, F. Naghdy, and H. Du, "Neural network-based passivity control of teleoperation system under time-varying delays," IEEE Transactions on Cybernetics, vol. 47, no. 7, pp. 1666-1680, 2016.

[372] J. Artigas, J.-H. Ryu, and C. Preusche, "Time domain passivity control for position-position teleoperation architectures," Presence: Teleoperators and Virtual Environments, vol. 19, no. 5, pp. 482-497, 2010.

[373] V. Chawda and K. Marcia, "Position synchronization in bilateral teleoperation under time-varying communication delays," IEEE/ASME Transactions on Mechatronics, vol. 20, no. 1, pp. 245-253, 2014.

[374] Y. Ye, Y.-J. Pan, and T. Hilliard, "Bilateral teleoperation with time-varying delay: a communication channel Passification approach," IEEE/ASME Transactions on Mechatronics, vol. 18, no. 4, pp. 1431-1434, 2013.

[375] I. Estrada-Sánchez, M. Velasco-Villa, and H. RodríguezCortés, "Prediction-based control for nonlinear systems with input delay," Mathematical Problems in Engineering, vol. 2017, Article ID 7415418, 2017.

[376] Z. Chen, F. Huang, C. Yang, and B. Yao, "Adaptive fuzzy backstepping control for stable nonlinear bilateral teleoperation manipulators with enhanced transparency 
performance," IEEE Transactions on industrial Electronics, vol. 67, no. 1, pp. 746-756, 2019.

[377] D.-H. Zhai and Y. Xia, "Adaptive fuzzy control of multilateral asymmetric teleoperation for coordinated multiple mobile manipulators," IEEE Transactions on Fuzzy Systems, vol. 24, no. 1, pp. 57-70, 2015.

[378] C. Yang, X. Wang, Z. Li, Y. Li, and C.-Yi Su, “Teleoperation control based on combination of wave variable and neural networks," IEEE Transactions on Systems, Man, and Cybernetics: Systems, vol. 47, no. 8, pp. 2125-2136, 2016.

[379] L. Zhao, L. Liu, Y. Wang, and H. Yang, "Active disturbance rejection control for teleoperation systems with actuator saturation," Asian Journal of Control, vol. 21, no. 2, pp. 702-713, 2019.

[380] L. Á. Castañeda, L. Guzman-Vargas, I. Chairez, and A. Luviano-Juárez, "Output based bilateral adaptive control of partially known robotic systems," Control Engineering Practice, vol. 98, Article ID 104362, 2020.

[381] Y. Xia, G. P. Liu, P. Shi, J. Han, and D. Rees, “Active disturbance rejection control for uncertain multivariable systems with time-delay," IET Control Theory \& Applications, vol. 1, no. 1, pp. 75-81, 2007.

[382] C. Join, M. Fliess, and F. Chaxel, "Model-free control as a service in the industrial internet of things: packet loss and latency issues via preliminary experiments," 2020, http:// arxiv.org/abs//2004.12156.

[383] Y. Yang, C. Hua, and X. Guan, “Adaptive fuzzy finite-time coordination control for networked nonlinear bilateral teleoperation system," IEEE Transactions on Fuzzy Systems, vol. 22, no. 3, pp. 631-641, 2013.

[384] Z. Li, X. Cao, and N. Ding, "Adaptive fuzzy control for synchronization of nonlinear teleoperators with stochastic time-varying communication delays," IEEE Transactions on Fuzzy Systems, vol. 19, no. 4, pp. 745-757, 2011.

[385] V. Lakshmikantham, V. M. Matrosov, and S. Sivasundaram, Vector Lyapunov Functions and Stability of Nonlinear Systems, Kluwer Academic Publishers, Berlin, Germany, 1st edition, 1991.

[386] V. I. Vorotnikov, Partial Stability and Control, Birkhåuser, New York, NY, USA, 1998.

[387] A. F. Filippov, Differential Equations with Discontinuities Right-Hand Sides, Kluwer Academic Publisher, Dordrecht, Netherlands, 1988.

[388] I. Podlubny, Fractional Differential Equations, Academic Press, San Diego, CA, USA, 1999.

[389] V. Utkin, "On convergence time and disturbance rejection of super-twisting control," IEEE Transactions on Automatic Control, vol. 58, no. 8, pp. 2013-2017, 2013.

[390] J. A. Moreno and M. Osorio, "Strict Lyapunov functions for the super-twisting algorithm," IEEE Transactions on Automatic Control, vol. 57, no. 4, pp. 1035-1040, 2012.

[391] A. E. Polyakov and A. S. Poznyak, "Method of Lyapunov functions for systems with higher-order sliding modes," Automation and Remote Control, vol. 72, no. 5, pp. 944-963, 2011.

[392] V. I. Utkin and A. S. Poznyak, "Adaptive sliding mode control with application to super-twist algorithm: equivalent control method," Automatica, vol. 49, no. 1, pp. 39-47, 2013.

[393] Y. Shtessel, C. Edwards, L. Fridman, and A. Levant, Sliding Mode Control and Observation, Birkhåuser, New York, NY, USA, 2014.

[394] P. Flandrin, "Wavelet analysis and synthesis of fractional Brownian motion," IEEE Transactions on Information Theory, vol. 38, no. 2, pp. 910-917, 1992.
[395] B. B. Mandelbrot and J. W. Van Ness, "Fractional Brownian motions, fractional noises and applications," SIAM Review, vol. 10, no. 2, pp. 422-437, 1968.

[396] B. B. Mandelbrot, "Broken line process derived as an approximation to fractional noise," Water Resources Research, vol. 8, no. 5, pp. 1354-1356, 1972.

[397] J. Humphrey, C. A. Schuler, and B. Rubinsky, "On the use of the Weierstrass-Mandelbrot function to describe the fractal component of turbulent velocity," Fluid Dynamics Research, vol. 9, no. 1-3, pp. 81-95, 1992.

[398] B. Ross, S. Samko, and E. Love, "Functions that have no first order derivative might have fractional derivatives of all order less than one," Real Analysis Exchange, vol. 20, no. 2, pp. 140-157, 1994.

[399] A. J. Muñoz-Vázquez, V. Parra-Vega, and A. Sánchez-Orta, "Continuous fractional-order sliding PI control for nonlinear systems subject to non-differentiable disturbances," Asian Journal of Control, vol. 19, no. 1, pp. 279-288, 2017.

[400] C. A. Monje, Y. Q. Chen, B. M. Vinagre, D. Xue, and V. Feliu-Batlle, Fractional-Order Systems and Controls: Fundamentals and Applications, Springer Science \& Business Media, Berlin, Germany, 2010.

[401] P. Shah and S. Agashe, "Review of fractional PID controller," Mechatronics, vol. 38, pp. 29-41, 2016.

[402] B. M. Vinagre, I. Podlubny, L. Dorcak, and V. Feliu, "On fractional PID controllers: a frequency domain approach," IFAC Proceedings Volumes, vol. 33, no. 4, pp. 51-56, 2000.

[403] D. Valério and J. S. da Costa, "Tuning of fractional PID controllers with Ziegler-Nichols-type rules," Signal Processing, vol. 86, no. 10, pp. 2771-2784, 2006.

[404] D. Valério and J. S. da Costa, "Tuning-rules for fractional PID controllers," in Proceedings of the Second IFAC Symposium on Fractional Differentiation and its Applications (FDA06), Porto, Portugal, July 2006.

[405] B. M. Vinagre, C. A. Monje, A. J. Calderón, and J. I. Suárez, "Fractional PID controllers for industry application: a brief introduction," Journal of Vibration and Control, vol. 13, no. 9-10, pp. 1419-1429, 2007.

[406] L. Liu, F. Pan, and D. Xue, "Variable-order fuzzy fractional PID controller," ISA Transactions, vol. 55, pp. 227-233, 2015.

[407] C. Hwang and Y.-C. Cheng, "A note on the use of the lambert $w$ function in the stability analysis of time-delay systems," Automatica, vol. 41, no. 11, pp. 1979-1985, 2005.

[408] C. Hwang and Y.-C. Cheng, "A numerical algorithm for stability testing of fractional delay systems," Automatica, vol. 42, no. 5, pp. 825-831, 2006.

[409] A. R. Fioravanti, C. Bonnet, H. Özbay, and S.-I. Niculescu, "A numerical method for stability windows and unstable root-locus calculation for linear fractional time-delay systems," Automatica, vol. 48, no. 11, pp. 2824-2830, 2012.

[410] C. Bonnet and J. R. Partington, "Coprime factorizations and stability of fractional differential systems," Systems \& Control Letters, vol. 41, no. 3, pp. 167-174, 2000.

[411] C. Bonnet and J. R. Partington, "Analysis of fractional delay systems of retarded and neutral type," Automatica, vol. 38, no. 7, pp. 1133-1138, 2002.

[412] J. R. Partington and C. Bonnet, " $\mathrm{H}_{\infty}$ and BIBO stabilization of delay systems of neutral type," Systems \& Control Letters, vol. 52, no. 3-4, pp. 283-288, 2004.

[413] C. Bonnet and J. R. Partington, "Stabilization of some fractional delay systems of neutral type," Automatica, vol. 43, no. 12, pp. 2047-2053, 2007.

[414] C. Bonnet, A. R. Fioravanti, and J. R. Partington, "Stability of neutral systems with commensurate delays and poles 
asymptotic to the imaginary axis," SIAM Journal on Control and Optimization, vol. 49, no. 2, pp. 498-516, 2011.

[415] C. Bonnet, "Stabilization of MISO fractional systems with delays," Automatica, vol. 83, pp. 337-344, 2017.

[416] D. Avanessoff, A. R. Fioravanti, and C. Bonnet, "YALTA: a Matlab toolbox for the $H_{\infty}$-stability analysis of classical and fractional systems with commensurate delays," IFAC Proceedings Volumes, vol. 46, no. 2, pp. 839-844, 2013.

[417] B. Dumitru, A. Ranjbar, S. J. Sadati, R. H. Delavari, and T. Abdeljawad, "Lyapunov-Krasovskii stability theorem for fractional systems with delay," Romanian Journal of Physics, vol. 56, no. 5-6, pp. 636-643, 2011.

[418] J.-B. Hu, G.-P. Lu, S.-B. Zhang, and L.-D. Zhao, "Lyapunov stability theorem about fractional system without and with delay," Communications in Nonlinear Science and Numerical Simulation, vol. 20, no. 3, pp. 905-913, 2015.

[419] W. Deng, C. Li, and J. Lü, "Stability analysis of linear fractional differential system with multiple time delays," Nonlinear Dynamics, vol. 48, no. 4, pp. 409-416, 2007.

[420] L. Chen, R. Wu, J. Cao, and J.-B. Liu, "Stability and synchronization of memristor-based fractional-order delayed neural networks," Neural Networks, vol. 71, pp. 37-44, 2015.

[421] H. Wang, Y. Yu, and G. Wen, "Stability analysis of fractionalorder Hopfield neural networks with time delays," Neural Networks, vol. 55, pp. 98-109, 2014.

[422] H. Wang, Y. Yu, G. Wen, S. Zhang, and J. Yu, "Global stability analysis of fractional-order Hopfield neural networks with time delay," Neurocomputing, vol. 154, pp. 15-23, 2015.

[423] G. Velmurugan, R. Rakkiyappan, and J. Cao, "Finite-time synchronization of fractional-order memristor-based neural networks with time delays," Neural Networks, vol. 73, pp. 36-46, 2016.

[424] S. Tyagi, S. Abbas, and M. Hafayed, "Global mittag-leffler stability of complex valued fractional-order neural network with discrete and distributed delays," Rendiconti del Circolo Matematico di Palermo Series 2, vol. 65, no. 3, pp. 485-505, 2016.

[425] V. Feliu-Batlle, R. Rivas Pérez, F. J. Castillo García, and L. Sanchez Rodriguez, "Smith predictor based robust fractional order control: application to water distribution in a main irrigation canal pool," Journal of Process Control, vol. 19, no. 3, pp. 506-519, 2009.

[426] V. Feliu-Batlle, R. Rivas-Perez, and F. J. Castillo-Garcia, "Fractional order controller robust to time delay variations for water distribution in an irrigation main canal pool," Computers and Electronics in Agriculture, vol. 69, no. 2, pp. 185-197, 2009.

[427] V. Feliu-Batlle, R. Rivas-Perez, and F. J. Castillo-García, "Simple fractional order controller combined with a smith predictor for temperature control in a steel slab reheating furnace," International Journal of Control, Automation and Systems, vol. 11, no. 3, pp. 533-544, 2013.

[428] T. N. Luan Vu and M. Lee, "Smith predictor based fractionalorder PI control for time-delay processes," Korean Journal of Chemical Engineering, vol. 31, no. 8, pp. 1321-1329, 2014.

[429] D. Boudjehem, M. Sedraoui, and B. Boudjehem, "A fractional model for robust fractional order Smith predictor," Nonlinear Dynamics, vol. 73, no. 3, pp. 1557-1563, 2013.

[430] C. I. Pop, C. Ionescu, R. De Keyser, and E. H. Dulf, "Robustness evaluation of fractional order control for varying time delay processes," Signal, Image and Video Processing, vol. 6, no. 3, pp. 453-461, 2012.
[431] P. Lanusse and A. Oustaloup, "Control of time-delay systems using robust fractional-order control and robust Smith predictor based control," in Proceedings of the ASME 2005 International Design Engineering Technical Conferences and Computers and Information in Engineering Conference, pp. 1475-1483, Long Beach, CA, USA, 2005.

[432] N. Maamri, M. Tenoutit, and J.-C. Trigeassou, "A comparison of fractional Smith predictors," in Proceedings of the 2013 European Control Conference (ECC), pp. 3991-3996, Zurich, Switzerland, July 2013. 